SOLUÇOES PERIOODICAS DE EQUAÇOES DIFERENCIAIS ALTONOMAS DO TIPO RETARDADO.

Wagner Vieira Leite Nunes 
SOLUÇJES PERIODDICAS DE EQUAÇOES DIFERENCIAIS

ALTONOMAS DO TIPO RETARDADO.

Wagner Vieira Leite Nunes

Orientador: Prof. Dr. Pläcido Zoēga Táboas

Dissertação apresentada ao Instituto de

Ciências Matemāticas de São Carlos, da Universidade de São Paulo, para a obtenção do Título de Mestre em Matemática. 
Dedico ao meu pai (in memorian)

a minha mãe,

\section{a Orā}

$e$ ags meus irmãos 


\section{AGRADECIMENTOS}

Agradeço ao Prof. Plácido Zoēga Täboas pela dedicação, incentivo e pelo exemplo de integridade profissional. Agradeço aos professores do Departamento de Matemática, do Instituto de Ciências Matemäticas de São Carlos, em particular aos professores Hildebrando Munhoz Rodrigues e José Gaspar Ruas Filho.

Agradeço aos professores do Departamento de Matemática, da Universidade Federal de são Carlos, principalmente ao professor Adalberto Panobianco Bergamasco pela dedicação nos tempos de graduação.

Agradeço a Lene pelo excelente trabalho de datilografia.

Este trabalho dependeu parcialmente de auxílios concedidos pela FAPESP, Proc.: 82/2002-0 e CNPq. 
This is a work on the existence of periodic solutions of Autonomous Functional Differential Equations of Retarded Type. The Hopf Bifurcation and applications of ejective points are done on basis of Hale's book [7]. A result is obtained for two-dimensional equations for wich $s^{l}$ is invariant. 
INTRODUÇAO $\ldots \ldots \ldots \ldots \ldots \ldots \ldots \ldots \ldots \ldots \ldots \ldots \ldots \ldots \ldots \ldots \ldots$

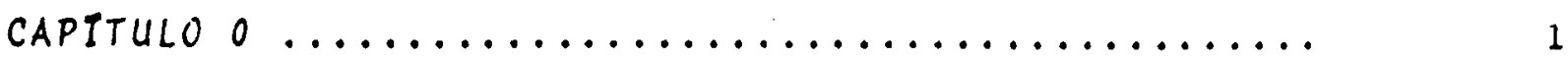

0.1 . O Teorema de Representação de Riesz ........... 1

0.2 . Teoremas de Ponto Fixo, Ejetividade ............ 2

0.3 . Teorema de Autovetor em Cones ............... 5

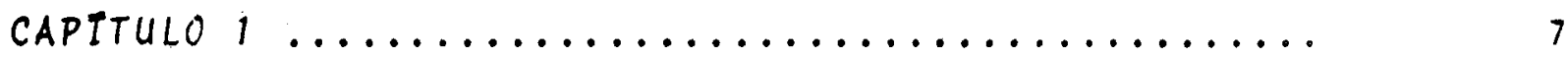

1.1. A Equação Diferencial Funcional com Retardamento. 7

1.2. A Equação Diferencial Funcional Linear com Retardamento .......................... 13

1.3. A Equação Diferencial Funcional Linear Autônoma com Retardamento ...................... 20

1.4. A Equação Linear Autônoma Forçada ........... 42

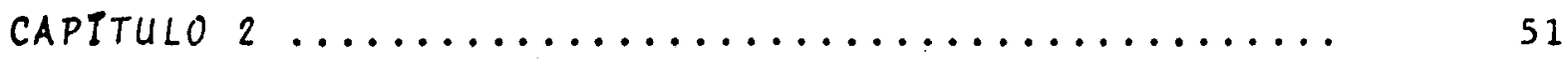

2.1. A Bifurcação de Hopt ................... 51

2.2. Teoremas sobre Periodicidade de EDFR Autōnomas Envolvendo Ponto Ejetivo ................. 60

2.3. Soluções Periōdicas de EDFR Autônomas em $c_{a} \ldots .64$

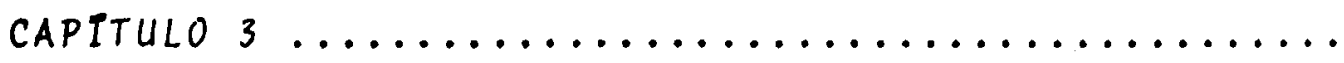




\section{INTRODUÇAO}

o objetivo principal deste trabalho é estudar o proble ma de existência de soluções periōdicas para Equações Diferenciais Funcionais Autônomas com Retardamento. Não tem pretensões de ineditismo e está fortemente baseado em [7]; no entanto, o resultado relativo a equações em $C\left([-r, 0], S^{1}(a)\right)$ e o Exemplo 3 têm inspiração em [2] e constituem contribuição original.

E apresentado em quatro capítulos.

No Capitulo zero citamos alguns resultados bäsicos para o desenvolvimento do trabalho, como o Teorema da Representa ção de Riesz, Teoremas envolvendo ponto ejetivo e um Teorema de autovetores em cones.

No Capitulo um definimos Equação Diferencial Funcional com Retardamento e apresentamos alguns resultados sobre existên cia, unicidade e dependência continua das soluções em relação as condições iniciais. Além disso, estudamos a Equação linear, em especial as Equações Lineares Autônomas.

o Capítulo dois é dedicado ao estudo da existência de soluções periódicas para Equações Autônomas. Neste capitulo en contram-se o Teorema da Bifurcação de Hopf, um teorema de perio dicidade de soluções envolvendo o conceito de ponto ejetivoe um Teorema sobre existência de soluções periödicas em $c\left([-r, 0], s^{1}(a)\right)$.

Por ültimo, no Capítulo três, utilizamos os resultados desenvolvidos no Capitulo dois em três exemplos. 
CAPITULO O

Apresentamos neste capitulo alguns resultados bảsicos que serão usados no decorrer do trabalho. As demonstrações podem ser encontradas na bibliografia indicada.

0.1. O Teorema de Representação de Riesz

Seja $C([a, b], R)$ o espaço das funções reais continuas em $[a, b]$, com a norma da convergência uniforme. Se $\mu \in \operatorname{VL}[a, b]$, conjunto das funções de variação limitada em [a,b], então funcional

$$
\begin{aligned}
C([a, b], R) & \rightarrow R \\
\psi & \longrightarrow \int_{a}^{b} d \mu(\theta) \psi(\theta)
\end{aligned}
$$

é linear e continuo. Vale a recíproca deste fato, para isto te$\operatorname{mos} 0:$

\section{Teorema 0.1 (Teorema de Representação de Riesz)}

Se $L: C([a, b], R) \rightarrow R \quad \bar{e}$ um funcional linear continuo, en tão existe $\mu \in V L[a, b]$ tal que para toda $\psi \in C([a, b], R)$ $L(\psi)=\int_{a}^{b} d \mu(\theta) \psi(\theta)$.

Uma demonstração deste fato pode ser encontrada em [10; pag. 413$]$.

Se $L: C([a, b], R) \rightarrow R^{n}$ é linear e contínua então existe uma matriz função $n(\theta), n \times n$, cujos termos são fun ções de variação limitada em $[a, b]$, tal que: 


$$
L(\psi)=\int_{a}^{b} \operatorname{dn}(\theta) \psi(\theta)
$$

para toda $\psi \in C\left([a, b], R^{n}\right)$.

\subsection{Teoremas de Ponto Fixo, Ejetividade}

Como uma conseqüência do Teorema de Ponto Fixo de Schau der temos o seguinte resultado: "Se $k$ è $u m$ subconjunto convexo, fechado e limitado de um espaso de Banach $\mathrm{X} e \mathrm{~A}: \mathrm{K} \rightarrow \mathrm{K}$ completamente continua então A tem um ponto fixo em $\mathrm{K}$."

A seguir enunciaremos alguns resultados de Roger D. Nussbaum, que nos levam a conclusões mais precisas com relação ao ponto fixo, isto é, daremos condições para garantir que o ponto fixo de A não seja um ponto prefixado, denominado ejetivo. As demonstrações desses resultados podem ser encontradas em [11].

\section{Definição 0.2}

Seja $K$ um conjunto convexo. Dizemos que $x_{0} \in K \quad \vec{e}$ um ponto extremo de $K$, se para todo $x_{1}, x_{2} \in K$ tal que $x_{0} \in\left\{t x_{1}+(1-t) x_{2}: 0 \leq t \leq 1\right\}$ tem-se $x_{0}=x_{1}$ ou $x_{0}=x_{2}$.

\section{$\underline{\text { Exemp 10 }}$}

Consideremos $K$ como sendo um n-cubo em $R^{n}$. Então os vértices do n-cubo serão os pontos extremos de $k$.

\section{Definição 0.3}

Seja $X$ um espaço de Banach, $U \subset x$ e $x_{0} \in U$. Dada uma aplicação $A: U \backslash\left\{x_{0}\right\} \rightarrow U$, o ponto $x_{0}$ é dito um ponto $e$ jetivo de $\mathrm{A}$, se existir uma vizinhança aberta de $x_{0}$, $G=G\left(x_{0}\right) \subset x$, tal que para todo $y \in G \cap U, y \neq x_{0}$, existe um 
$m=m(y) \in N$ tal que $A^{m} y \notin G \cap U$.

\section{Exemplos}

$\left(1 .{ }^{\circ}\right)$ Consideremos $U=X=R$ e $A: R \backslash\{0\} \rightarrow R$ dada por $A(x)=1 / x$. Então $x_{0}=0$ é um ponto ejetivo de $A$, pois tomando-se $G=G(0)=(-1,1)$ segue que para $y \in G \backslash\{0\}$, Ay $\notin G$, logo basta tomar $m=1$.

A vizinhança, $G$, neste caso, pode ser qualquer inter valo aberto contido em $(-1,1)$ que contenha $x_{0}=0$.

$\left(2 .^{\circ}\right)$ seja $X$ um espaço de Banach, $U=X$ e $A: X \backslash\{0\} \rightarrow X$ dada por $A u=u+1 / 2(u-u /\|u\|)$, para $u \in X \backslash\{0\}$. Então $x_{0}=0$ é um ponto ejetivo de A.

Para todo $M>0$, sejam

$S_{M}=\{x \in X:|x|=M\} \quad$ e $\quad B_{M}=\{x \in X:|x| \leq M\}$.

Daremos a seguir dois resultados que darão condições pa ra que um ponto fixo de uma aplicação não ocorra num ponto ejetivo da mesma.

\section{$\underline{\text { Teorema } 0.4}$}

Seja $X$ um espaço do Banach e $K \subset x$, limitado, fe chado, convexo e de dimensão infinita. Se $A: K \backslash\left\{x_{0}\right\} \rightarrow K \bar{e}$ completamente contínua, $x_{0} \in K \vec{e} u m$ ponto ejetivo de A então existe um ponto fixo de $A$ em $K \backslash\left\{x_{0}\right\}$. se $K \ddot{e} d e$ di mensãofinita $e x_{0} \bar{e} u m$ ponto extremo de $k$, podemos concluir - mesmo.

Observemos que se $K$ è de dimensão finita, basta que a aplicação A seja contínua; $x_{0}$ ser um ponto ejetivo é con- 
dição essencial nas hipóteses do Teorema 0.4 como mostra o se guinte exemplo.

\section{Exemplo}

Seja $X=R^{2}, \quad K$ otriângulo comvërtices $(1,0),(0,0)$

e $(0,1)$ e $A: K \backslash\{0\} \rightarrow K$ dada por $A u=u / 2$.

o conjunto $K$ e a aplicação A satisfazem as hipóteses do Teorema 0.4 , porém não existe um ponto fixo em $k \backslash\{0\}$. Isto ocorre porque $x_{0}=0$ não é um ponto ejetivo de A.

\section{Teorema 0.5}

Seja $X$ um espaço de Banach e $K \subset x$, convexo e fechado. Se $A: K \backslash\{0\} \rightarrow K$ é completamente continua, $0 \in K \bar{e}$ um ponto ejetivo de $A$ e existe $M$. > 0 tal que se $A x=\lambda x$, $p \underline{a}$ ra $x \in K \cap S_{M}$ implicar $\lambda<1$ então A tem um ponto fixo em $K \backslash\{0\}$ se $K$ é de dimensão infinita ou se 0 é um ponto extremo de $K$ e $K$ é de dimensãofinita.

observemos que no caso de $K$ ser de dimensãofinita a condição do ponto ejetivo ser extremo é essencial para que as conclusões 0.4 e 0.5 ocorram, como mostra o exemplo a seguir:

\section{Exemp 10}

Seja $\quad X=R^{2}, \quad K=B_{1}(0)$ e $x_{0}=0$. Se $R=\left[\begin{array}{cc}\cos \pi / 4 & -\operatorname{sen} \pi / 4 \\ \operatorname{sen} \pi / 4 & \cos \pi / 4\end{array}\right]$ e $\quad S x=x /|x| ;$ definindo $A: K \backslash\{0\} \rightarrow K$ por $A x=R S x$.

A aplicação A, projeta o vetor $x$ sobre a esfera unitária e aplica-lhe uma rotação de um ângulo de $\pi / 4$. Todas as 
hipöteses do Teorema 0.5 estão satisfeitas, exceto de $x_{0}=0$ ser um ponto extremo de $K$ e a aplicação $A$ não tem um ponto fixo em $K \backslash\{0\}$.

\section{3 . Teorema de Autovetor em Cones}

Nesta seção, daremos um teorema que nos garantiráa xistência de um autovetor de uma aplicação completamente conti nua em um cone. A demonstração deste resultado pode ser encontrada em $[6 ; \mathrm{pag}, 144]$.

Para isto necessitaremos de algumas definições.

Definição 0.6

Seja E um espaço de Banach. Um conjunto $K \subset E$ è de nominado um cone, se satisfaz:

(i) K é fechado e convexo.

(ii) Se $\phi \in K$ então $\lambda \phi \in K$, para todo $\lambda \geq 0$.

(iii) Para toda $\phi \in E, \phi \neq 0$, se $\phi \in K$ então $-\phi \notin K$.

\section{Exemplos}

$\left(1 .^{\circ}\right)$ Seja $E=R^{2}, k_{1}<k_{2}$, então o conjunto

$K=\left\{x=\left(x_{1}, x_{2}\right) \in R^{2}: 0 \leq k_{1} x_{1} \leq x_{2} \leq k_{2} x_{1}\right\}$

é um cone em $R^{2}$.

$\left(2 .^{\circ}\right)$ seja $E=C\left([-r, 0], R^{2}\right)$ onde $r>0$, e $k_{1}<k_{2}$, então o conjunto,

$K=\left\{\phi=\left[\begin{array}{l}\phi_{1} \\ \phi_{2}\end{array}\right] \in E: 0 \leq k_{1} \phi_{1}(\theta) \leq \phi_{2}(\theta) \leq k_{2} \phi_{1}(\theta)\right.$, para $\left.-r \leq \theta \leq 0\right\}$. $\vec{e}$ um cone em $C\left([-r, 0], R^{2}\right)$. 
Definição 0.7

Dizemos que $K \subset E$ è um cone truncado, se $K \vec{e}$ a in tersecção de um cone com uma vizinhança conexa de zero.

\section{Definição 0.8}

Dizemos que uma aplicação $T: K \rightarrow K$, linear ou. não, tem um autovetor $\phi \in K$, se existir uma constante $\mu$, tal que $\mathrm{T} \phi=\mu \phi$.

Com is to temos o:

\section{Teorema 0.9}

Sejam $K \subset E$ um cone ou cone truncado, onde $E \vec{e}$ um espaço de Banach e $\partial G$, a fronteira de um conjunto aberto limitado $G \subset E$, com $0 \in G$. Se $T: \partial G n K \rightarrow K$ é completamen te continua e

$$
\inf \{|\mathrm{T} \phi|: \phi \in \partial G \cap K\}>0
$$

então $T$ tem um autovetor em $\partial G \cap K$. 


\section{CAPITULO}

\subsection{A Equação Diferencial Funcional com Retardamento}

Seja $R=(-\infty, \infty), R^{n}$ o espaço vetorial n-dimensional com uma norma fixada, 1.1 , e $[a, b]$ um intervalo fechado da reta.

Consideremos $C\left([a, b], R^{n}\right)$ o espaço de Banach das Eunçöes. $\phi:[a, b] \rightarrow R^{n}$ continuas, munido da seguinte norma

$$
|| \phi||=\sup _{a \leq t \leq b}|\phi(t)| .
$$

Seja $r>0$, um nümero real fixo. Se $[a, b]=[-r, 0]$, denotaremos por $C=C\left([-r, 0], R^{n}\right)$.

Se $\sigma \in R, A \geq 0$ e $\quad x \dot{\epsilon} C\left([\sigma-r, \sigma+A], R^{n}\right)$ então para todo $t \in[\sigma, \sigma+A]$, seja $x_{t} \in C$ definida por

$$
x_{t}(\theta)=x(t+\theta) \quad \text { onde }-r \leq \theta \leq 0 \text {. }
$$

Se $D \subset R \times C, f: D \rightarrow R^{n}$ é uma função dada e "." re presenta a derivada à direita com relação a $t$, dizemos que a re 1 ação

$$
\text { (1.1) } \quad \dot{x}(t)=f\left(t, x_{t}\right)
$$

é uma equagão diferencial funcional com retardamento em $\mathrm{D}$ e se rā denotada por EDFR. Se desejarmos enfatizar que a equação é de finida por f, nōs escreveremos EDFR(f).

A equação (1.1) inclui como caso particular as equações diferenciais ordinärias, basta para isto tomar $r=0$. 


\section{Exemplos}

$\left(1 .^{\circ}\right)$ A equação

$\dot{x}(t)=g(t, x(t-1))$

é uma EDFR. Neste caso $r=1$ e $f(t, \phi)=g(t, \phi(-1))$, pa$r a(t, \phi) \in R \times C$.

$\left(2 .^{\circ}\right)$ A equação

$\dot{x}(t)=g\left(t, x(t), x\left(t-h_{1}(t)\right), \ldots, x\left(t-h_{m}(t)\right)\right)$

onde $0 \leq h_{j}(t) \leq r<\infty$, para $j=1, \ldots, m, \quad$ è uma EDFR. Neste caso

$f(t, \phi)=g\left(t, \phi(0), \phi\left(-h_{1}(t)\right), \ldots, \phi\left(-h_{m}(t)\right)\right)$,

para $(t, \phi) \in R \times C$.

$\left(3 .^{\circ}\right)$ A equação

$\dot{x}(t)=\int_{-r}^{0} g(t, \theta, x(t+\theta)) d \theta$

é uma EDFR. Neste caso $f(t, \phi)=\int_{-r}^{0} g(t, \theta, \phi(\theta)) d \theta$ para $(t, \phi) \in R \times C$.

os exemplos acima foram extraídos de [12; pag. 4 e 5].

\section{Definição 1.1}

Dizemos que uma função $x$ é solusão da equagão (1.1) em $[\sigma-r, \sigma+A)$ se existem $\sigma \in R$ e $A>0$ tal que:

(i) $x \in c\left([\sigma-r, \sigma+A), R^{n}\right)$

(ii) $\left(t, x_{t}\right) \in D$ e $x(t)$ satisfaz a equação (1.1) para $\mathbf{t} \in[\sigma, \sigma+A)$.

Dado $(\sigma, \phi) \in D$, dizemos que $x(\sigma, \phi, f)$ é uma solucão da equagão (1.1) passando por $(\sigma, \phi)$, se existir A > 0 tal que: 
(i) $x(\sigma, \phi, f)$ é solução da equação (1.1) em $[\sigma-r, \sigma+A)$.

(i i) $\mathbf{x}_{\sigma}(\sigma, \phi, f)=\phi$.

\section{Exemplo}

Consideremos a equação

$$
\dot{x}(t)=e^{r} x(t-r) \quad \text { (onde } r>0 \text { ) }
$$

então uma solução da equação em $[-r, \infty)$ passando por $(0, \phi)$ on de $\phi=1 \quad \vec{e}$

$$
x(t)= \begin{cases}1 & \text { se }-r \leq t \leq 0 \\ e^{t} & \text { se } t>0\end{cases}
$$

\section{Definições 1.2}

Dizemos que a equação ( 1.1$)$ è Zinear se $f(t, \phi)=L(t, \phi)+h(t)$ para $(t, \phi) \in D$ onde $L(t, \phi)$ é linear em $\phi$ para cada $t \in[\sigma, \sigma+A)$. Dizemos que a equação (1.1) è autônoma se $f(t, \phi)=g(\phi)$, onde $g$ não depende de $t$.

Observemos que encontrar uma solução da equação (1.1) pas sando por $(\sigma, \phi) \in D, \operatorname{com} f: D \rightarrow R^{n}$ continua é equivalente a resolver a equação integral

$$
\text { (1.2) } \quad x(t)=\phi(0)+\int_{\sigma}^{t} f\left(s, x_{s}\right) d s, \quad t \geq \sigma \text {. }
$$

Apresentaremos a seguir alguns resultados sobre existẹn cia, unicidade, dependência continua em relação aos dados iniciais, das soluções da equação (1.1). As demonstrações destes re sultados podem ser encontradas em $[7 ;$ pag. 41, 42].

Se $V C R \times C$, definimos $C^{0}\left(V, R^{n}\right)$ como sendo o espaço de Banach das funções continuas e limitadas de $V$ em $R^{n}$, mu 
nido da norma

$$
|f|_{V}=\sup _{(t, \phi) \in V}|f(t, \phi)|
$$

\section{Teorema 1.3 (Existência)}

Suponhamos que $\Omega \subset R \times C$ e aberto e que $f^{0} \in C\left(\Omega, R^{n}\right)$. Se $(\sigma, \phi) \in \Omega$ então existe uma solução da $\operatorname{EDFR}\left(f^{\circ}\right)$ passando por $(\sigma, \phi)$. Em geral, se $W \subset \Omega$ é compacto e $f^{0} \in C\left(\Omega, R^{n}\right)$ são dados então existe uma vizinhança de $W, V=V(W)<\Omega$ tal que $\mathrm{f}^{0} \in \mathrm{C}^{0}\left(\mathrm{~V}, \mathrm{R}^{\mathrm{n}}\right)$, existe uma vizinhança de $\mathrm{f}^{0}, U=U\left(f^{0}\right) \subset C^{0}\left(V, R^{n}\right)$ e $\alpha>0$ tal que, para todo $(\sigma, \phi) \in W$ e $f \in U$, existe uma solução $x(\sigma, \phi, f)$ da EDFR(f) passando por $(\sigma, \phi)$ que existe em $[\sigma-r, \sigma+\alpha]$.

Teorema 1.4 (Continuidade em relação às condições iniciais)

Suponhamos que $\Omega \subset R \times C$ è aberto, $\left(\sigma^{\circ}, \phi^{\circ}\right) \in \Omega$, $f^{\circ}$ e $C\left(\Omega, R^{n}\right)$ e que $x^{\circ}$ é uma solução da EDFR( $\left.{ }^{\circ}\right)$ passando por $\left(\sigma^{\circ}, \phi^{\circ}\right)$ que existe e é ünica em $\left[\sigma^{\circ}-r, b\right]$. Seja $w^{0} \subset \Omega$ o con junto compacto, definido por:

$$
W^{0}=\left\{\left(t, x_{t}^{0}\right): t \in\left[\sigma^{0}, b\right]\right\}
$$

e seja $V^{\circ}$ uma vizinhança de $W^{0}$ tal que $f^{0} \in C^{0}\left(V^{0}, R^{n}\right)$. Se $\left(\sigma^{k}, \phi^{k}, f^{k}\right)$ para $k=1,2, \ldots$ satisfaz $\sigma^{k} \rightarrow \sigma^{\circ}, \phi^{k} \rightarrow \phi^{\circ}$ e $\left|f^{k}-f^{0}\right|_{V^{\circ}} \rightarrow 0$ quando $k \rightarrow \infty$, então existe um $k^{0} \in N, \quad$ onde $\operatorname{EDFR}\left(\mathrm{f}^{\mathrm{k}}\right)$, para $k \geq k^{0}$, è tal que, cada solução $x^{k}=x^{k}\left(\sigma^{k}, \phi^{k}, f^{k}\right)$ passando por $\left(\sigma^{k}, \phi^{k}\right)$ existe em $\left[\sigma^{k}-r, b\right]$ e $x^{k}+x^{o}$ unifor memente em $\left[\sigma^{\circ}-r, b\right]$. Como todas as $x^{k}$ podem não estar definidas em $\left[\sigma^{\circ}-r, b\right]$, por $x^{k} \rightarrow x^{0}$ uniformemente em $\left[\sigma^{\circ}-r, b\right]$, nós queremos dizer que: para todo $\varepsilon>0$, existe $k_{1}=k_{1}(\varepsilon)$ tal que se $k \geq k_{1}, x^{k}(t)$ estä definida em $\left[\sigma^{0}-r-\varepsilon, b\right]$ e 
$x^{k} \rightarrow x^{\circ}$ uniformemente em $\left[\sigma^{\circ}-r-\varepsilon, b\right]$.

\section{Definição 1.5}

Seja $\Omega \subset R \times C$ aberto e $f \in C\left(\Omega, R^{n}\right)$. Dizemos que $f(t, \phi)$ é Zocalmente Lipschitziana com relaşão a $\phi$ em $\Omega$ se f é lipschitziana em $\phi$ para cada subconjunto compacto de $\Omega$, isto é, se $W \subset \Omega$ é compacto, existe $k=k(W) \in R$ tal que pa ra todo $(t, \phi),(t, \psi) \in W$ temos

$$
|f(t, \phi)-f(t, \psi)| \leq k|\phi-\psi|
$$

\section{Teorema 1.6 (Unicidade)}

Seja $\Omega \subset R \times C$ aberto e $f \in C\left(\Omega, R^{n}\right)$ localmentelips chitziana com relação a $\phi$ em $\Omega$. Se $(\sigma, \phi) \in \Omega$ então exis te uma ünica solução da $\operatorname{EDFR}(f)$ passando por $(\sigma, \phi)$.

\section{Definição 1.7}

Suponhamos que $f$ da equação (1.1) é continua. Se $x$ é uma solução da EDFR(f) no intervalo $[\sigma, a), a>\sigma$, dizemos que uma função $\hat{x}$ é uma continuação (a direita) de $x$, se existe $b>a$, tal que $\hat{x}$ está definida em $[\sigma-r, b)$, coincide $\operatorname{com} x$ em $[\sigma-r, a)$ e satisfaz a equação (1.1) em $[\sigma, b)$.

Dizemos que $x$, solução da equação $(1.1)$ em $[\sigma, a), \vec{e}$ não continuável (a direita) se não existe uma continuação dela, neste caso o intervalo $[\sigma, a)$ è dito intervalo maximal de existência da solução.

\section{Observações 1.8}

(1) Colocando-se algumas condições sobre a EDFR(f), podemos obter resultados importantes sobre continuação de 
soluções e soluções não continuáveis de uma EDFR(f). Para maiores detalhes indicamos [7; secção 2.3].

(2) Se a equação (1.1) for $\mathrm{T}$-periódica, isto é, $\mathrm{f}(\mathrm{t}+\mathrm{T}, \phi)=$ $=f(t, \phi), \quad t \in R$, uma solução T-periōdica, implicitą mente, envolve a consideração do intervalo $J=(-\infty, \infty)$ como seu domínio. Isto segue do fato de $x(t)$ ser solução T-periódica da equação (1.1) implicar $x(t+T)$ tambēm è solução T-periódica da equação (1.1).

\section{Definição 1.9}

Suponhamos que $\mathrm{f} \in \mathrm{C}\left(\Omega, \mathrm{R}^{\mathrm{n}}\right)$, onde $\Omega \subset \mathrm{R} \times \mathrm{C}$. Para todo $(\sigma, \phi) \in R \times C$ existe um $t_{\sigma, \phi} \in R$ e uma função $x$ que $\bar{e}$ uma solução não continuāvel da EDFR(f) passando por $(\sigma, \phi)$ com intervalo maximal de existência $\left[\sigma-r, t_{\sigma, \phi}\right)$. seja $\Omega_{\sigma} \subset \mathrm{C}$ dado por $\Omega_{\sigma}=\{\phi \in C:(\sigma, \phi) \in \Omega\}$; definimos a aplicação

$$
\begin{aligned}
T(t, \sigma): & \Omega_{\sigma} \rightarrow \mathrm{C} \\
\phi & \rightarrow \mathrm{x}_{t}(\sigma, \phi)
\end{aligned} \quad \text { para } t \in\left[\sigma, t_{\sigma, \phi}\right)
$$

denominada aplicação solução associada a EDFR(f).

\section{Observações 1.10}

(1) A aplicação solução associada a uma EDFR possui, em alguns casos, propriedades indesejāveis, como, por exemplo: não ser limitada, isto é, não leva conjuntos limitado e fechado em conjuntos limitado; não ser injetora. Para obter mais informações sobre estes fatos ver [7; cap. 3]. 
(2) Colocando-se algumas condições sobre a EDFR podemos ga rantir que a aplicação solução será injetora. Veja [7; seç̧ão 2.5$]$.

\section{Definição 1.11}

Dada uma EDFR(f), dizemos que um subconjunto B $\subset \Omega_{\sigma}$ é invariante pela aplicasão solusão ou B é $\mathrm{T}(\mathrm{t}, \sigma)$-invariante se $T(t, \sigma) B \subset B$ para $t \in\left[\sigma, t_{\sigma, \phi}\right)$.

\section{Exemp10}

Consideremos

$\left\{\begin{array}{l}\dot{x}(t)=-x(t-1) y(t)-z(t) \\ \dot{y}(t)=x(t-1) x(t)-z(t) \\ \dot{z}(t)=x(t)+y(t)\end{array}\right.$

As soluções deste sistema satisfazem:

$x(t) \dot{x}(t)+y(t) \dot{y}(t)+z(t) \dot{z}(t)=0$, is to e,,$x^{2}(t)+y^{2}(t)+z^{2}(t)=a^{2}$,

$t \geq 0$, para a $>0$ qualquer. Logose $\phi=\left(\phi_{1}, \phi_{2}, \phi_{3}\right) \in C\left([-1,0], R^{3}\right) \vec{e}$ tal que $\phi \in C_{a}=C\left([-1,0], s^{2}(a)\right)$ onde $s^{2}(a)=\left\{x \in R^{3}:|x|=a\right\}$ então temos que a solução $x(0, \phi)=(x(0, \phi), y(0, \phi), z(0, \phi))$ do sistema acima permanecera em $s^{2}(a), \log 0 \mathrm{~T}(t, 0) \phi \in \mathrm{C}_{a}, t \geq 0$ se $\phi \in C_{a}$, mostrando que $C_{a}$ é invariante pela aplicação solução $T(t, 0)$, para $t \geq 0$.

o exemplo acima foi extraido de [8].

\subsection{A Equação Diferencial Funcional Linear com Retardamento}

Para $(\sigma, \phi)$ e $\times C$, consideremos o sistema linear não homogēneo, 
$(1.3)$

$$
\dot{x}(t)=L\left(t, x_{t}\right)+h(t), \quad t \geq 0
$$

$$
x_{\sigma}=\phi
$$

e o sistema linear homogêneo,

$(1.4) \quad \dot{x}(t)=L\left(t, x_{t}\right), \quad t \geq 0$

onde $L(t, \phi)$ é linear em $\phi$, continua em $(t, \phi)$ e $h \in C\left([\sigma, \infty), R^{n}\right)$.

Suponhamos que existe uma função $m \in L_{1}^{10 c}(R, R)$, o es paço das funções lebesgue integrāveis em cada intervalo compacto de R, tal que,

(1.5) $\quad|L(t, \phi)| \leq m(t)|\phi|$.

para $t \in R$ e $\phi \in C$. De acordo com o Teorema de Riesz, podemos considerar uma matriz $\eta=n(t, \theta), n \times n$, de variação $1 i-$ mitada em $\theta$, para cada $t$ fixado, $\operatorname{com} n(t, \theta)=0$ para $\theta \geq 0$ e $\eta(t, \theta)=\eta(t,-r)$ para $\theta \leq-r$, tal que

$$
L(t, \phi)=\int_{-r}^{0} d_{\theta} \eta(t, \theta) \phi(\theta)
$$

para $(t, \phi) \in R \times C$.

Comestas hipóteses, temos que para cada $(\sigma, \phi) \in \mathrm{R} \times \mathrm{C}$, a equação (1.3) tem uma única solução passando por $(\sigma, \phi)$ que é continua em $[\sigma-r, \infty)$, que serä denotada por $x(\sigma, \phi, h)$.

Se $x(\sigma, \phi, h)$ é a solução da equação (1.3) passando por $(\sigma, \phi)$ então da linearidade de L e da unicidade das soluções, segue que

$$
x(\sigma, \phi, h)=x(\sigma, \phi, 0)+x(\sigma, 0, h)
$$


$(1.6 . a) \quad|x(\sigma, \phi, 0)(t)| \leq|\phi| \exp \int_{\sigma}^{t} m(s) d s$

(1.6.b) $\quad|x(\sigma, 0, h)(t)| \leq \int_{\sigma}^{t}|h(s)| d s \exp \int_{\sigma}^{t} m(s) d s$, para $t \geq \sigma$.

Logo, $x(\sigma, \phi, 0)$ e $x(\sigma, 0, h)$ são lineares e continuas

em $\phi e$, respectivamente.

o resultado que segue, nos dá uma representação da solução $x(\sigma, \phi, h)$ em termos de uma matriz $U(t, s)$.

Teorema 1.12 (Fórmula da Variação das Constantes)

Se $h \in L_{1}^{10 c}\left([\sigma, \infty), R^{n}\right)$, L satisfaz as condições acima e $x(\sigma, \phi, h)$ è uma solução da equação (1.3) então

$$
x(\sigma, \phi, h)(t)=x(\sigma, \phi, 0)(t)+\int_{\sigma}^{t} U(t, s) h(s) d s, \quad t \geq \sigma
$$

$$
x_{\sigma}=\phi
$$

onde $U(t, s)$ é a solução da equação

(1.8) $U(t, s)=\left\{\begin{array}{l}\int_{s}^{t} L\left(u, U_{U}(\cdot, s)\right) d u+I \text {. em } s, t \geq s \\ 0.5 \text { se } s-r \leq t \leq s\end{array}\right.$

ou

(1.9) $\frac{\partial U(t, s)}{\partial t}=L\left(t, U_{t}(\cdot, s)\right), t \geq s, q . s$. em s e $t$

$$
U(t, s)= \begin{cases}0, & s-r \leq t<s \\ I, & t=s\end{cases}
$$

onde $U_{t}(\cdot, s)(\theta)=U(t+\theta, s)$ para $-r \leq \theta \leq 0$.

A função $U(t, s)$ é chamada matriz fundamentaz. 


\section{Corolário 1.13}

Se a equação $(1.4)$ é autônoma então $U(t, s)=U(t-s, 0)=$ $d \underline{\underline{E}} U(t-s) \quad e$

(1.10)

$$
x(\sigma, \phi, h)(t)=x(t-\sigma, \phi, 0)(0)+\int_{\sigma}^{t} U(t-s) h(s) d s, t \geq \sigma
$$

$$
x_{\sigma}=\phi
$$

\section{Demonstração}

Temos que, $U(t, s)=U(t-s, 0)$ para $s-r \leq t \leq s$ e pa ra $t \geq s$, segue de $(1.9)$ que

$$
\frac{\partial U(t-s, 0)}{\partial t}=L\left(U_{t-s}(\cdot, 0)\right) \text {. }
$$

Portanto, $U(t-s, 0)$ é solução de (1.9) para $t-s \geq 0$, que coincide com $U(t, s)$ para $s-r \leq t \leq s$, então pela unicida de segue que $U(t-s, 0)=U(t, s)$ para $t \geq s$.

Usando a fórmula da variação das constantes e o resulta do provado acima, vemos que para provar (1.10) basta mostrar que $x(\sigma, \phi, 0)(t)=x(t-\sigma, \phi, 0)(0)$.

Esta igualdade segue do fato da equação ser autônoma.

A aplicação solução associada a equação (1.4) dada por

(1.11) $\quad T(t, \sigma) \phi=x_{t}(\sigma, \phi, 0)$

será um operador linear, contínuo e vale a seguinte relação:

$$
U_{t}(\cdot, s)=T(t, s) x_{0}
$$

(1.12) onde

$$
X_{0}(\theta)= \begin{cases}0 & \text { se }-r \leq \theta<0 \\ I & \text { se } \theta=0\end{cases}
$$


Logo teremos,

(1.13) $x_{t}(\sigma, \phi, h)=T(t, \sigma) \phi+\int_{\sigma}^{t} T(t, s) x_{0} h(s) d s, t \geq \sigma$.

o próximo resultado nos dā uma representação de

$x(\sigma, \phi, 0)(t)$ em termos da matriz solução da equação adjunta formar:

$$
y(s)+\int_{s}^{\infty} y(\alpha) n(\alpha, s-\alpha) d \alpha=c t e
$$

onde $y \in R^{n^{*}}$, o espaço $n$-dimensional dos vetores linha.

\section{Teorema.1.14.}

Se $x$ é solução da equação (1.3) em $[\sigma, \infty)$ passando por $\left(\sigma, x_{\sigma}\right)$ então para todo $t \geq \sigma$,

$$
\begin{aligned}
x(t) & =Y(\sigma, t) x(\sigma)+ \\
& +\int_{\sigma-r}^{\sigma} d \alpha\left\{\int_{\sigma}^{t} Y(s, t) \eta(s, \alpha-s) d s\right\} x(\alpha)+\int_{\sigma}^{t} Y(s, t) h(s) d s
\end{aligned}
$$

onde a matriz $Y, n \times n$ é definida por

(1.15) $\quad Y(\sigma, t)=\left\{\begin{array}{l}0, t<\sigma \\ I-\int_{\sigma}^{t} Y(\alpha, t) n(\alpha, \sigma-\alpha) d \alpha, \quad t \geq \sigma\end{array}\right.$

Além disso, $Y(\sigma, t)=U(t, \sigma)$ q.s. em $\sigma$.

\section{Demonstração}

Temos que

(1.16) $\dot{x}(t)=L\left(t, x_{t}\right)+h(t)=\int_{-r}^{0} d_{\theta} n(t, \theta) x(t+\theta)+h(t), t \geq \sigma$ e fazendo a mudança $t+\theta=s$, obtemos 
$(1.17) \quad \dot{x}(t)=\int_{t-r}^{t} d s \eta(t, s-t) x(s)+h(t), \quad t \geq 0$.

Seja $Y(\sigma, t)$ dada por $(1.15)$.

Como

$\int_{\sigma}^{t} d_{S} Y(s, t) x(s)=\left.Y(s, t) x(s)\right|_{\sigma} ^{t}-\int_{\sigma}^{t} Y(s, t) \dot{X}(s) d s$

$=X(t)-Y(\sigma, t) X(\sigma)-\int_{\sigma}^{t} Y(s, t) \dot{X}(s) d s$.

Logo,

$X(t)=Y(\sigma, t) x(\sigma)+\int_{\sigma}^{t} d_{s} Y(s, t) x(s)+\int_{\sigma}^{t} Y(s, t) \dot{X}(s) d s$.

Substituindo-se $\dot{x}(s)$ por (1.17), obtemos:

$(1.18) \quad x(t)=Y(\sigma, t) x(\sigma)+\int_{\sigma}^{t} d_{s} Y(s, t) x(s)+$

$$
+\int_{\sigma}^{t} Y(s, t)\left[\int_{s-r}^{s} d \alpha^{n(s, \alpha-s) x(\alpha)] d s}\right.
$$

Definindo-se $p(t, s)=\eta(t, s-t)$, a ültima parcela do segundo membro de $(1.18)$, torna-se:

$$
\int_{\sigma}^{t} Y(s, t)\left[\int_{s-r}^{s} d_{\alpha} p(s, \alpha) x(\alpha)\right] d s
$$

Como para $\alpha<s-r$ temos $p(s, \alpha)=$ cte e para $s<\alpha$ $p(s, \alpha)=0$, temos que $d_{\alpha} p(s, \alpha)=0$ para $\sigma-r<\alpha<s-r$ e para $s<\alpha<t, 10 g 0$

$$
\begin{aligned}
& \int_{\sigma}^{t} Y(s, t)\left[\int_{s-r}^{s} d \alpha p(s, \alpha) x(\alpha)\right] d s= \\
= & \int_{\sigma}^{t} Y(s, t)\left[\int_{\sigma-r}^{t} d_{\alpha} p(s, \alpha) x(\alpha)\right] d s
\end{aligned}
$$




$$
\begin{aligned}
\text { Usando }[4] \text { temos } & \int_{\sigma}^{t} Y(s, t)\left[\int_{s-r}^{s} d_{\alpha} p(s, \alpha) x(\alpha)\right] d s= \\
= & \int_{\sigma-r}^{t} d_{\alpha}\left(\int_{\sigma}^{t} Y(s, t) p(s, \alpha) d s\right) x(\alpha)= \\
= & \int_{\sigma-r}^{\sigma} d \alpha\left(\int_{\sigma}^{t} Y(s, t) p(s, \alpha) d s\right) \times(\alpha)+ \\
+ & \int_{\sigma}^{t} d_{\alpha}\left(\int_{\sigma}^{t} Y(s, t) p(s, \alpha) d s\right) x(\alpha)
\end{aligned}
$$

Substituindo em ( 1.18$)$, teremos

$$
\begin{aligned}
& X(t)=Y(\sigma, t) x(\sigma)+\int_{\sigma}^{t} d_{\alpha} Y(\alpha, t) x(\alpha)+\int_{\sigma}^{t} Y(s, t) h(s) d s+ \\
& +\int_{\sigma-r}^{\sigma} d_{\alpha}\left(\int_{\sigma}^{t} Y(s, t) n(s, \alpha-s) d s\right) x(\alpha)+ \\
& +\int_{\sigma}^{t} d_{\alpha}\left(\int_{\sigma}^{t} Y(s, t) n(s, \alpha-s) d s\right) x(\alpha)= \\
& =Y(\sigma, t) x(\sigma)+\int_{\sigma}^{t} d_{\alpha} Y(\alpha, t) x(\alpha)+\int_{\sigma}^{t} Y(s, t) h(s) d s+ \\
& +\int_{\sigma-r}^{\sigma} d \alpha\left(\int_{\sigma}^{t} Y(s, t) \eta(s, \alpha-s) d s\right) x(\alpha)+ \\
& +\int_{\sigma}^{t} d_{\alpha}\left(\int_{\alpha}^{t} Y(s, t) n(s, \alpha-s) d s\right) x(\alpha)= \\
& =Y(\sigma, t) x(\sigma)+\int_{\sigma}^{t} Y(s, t) h(s) d s+ \\
& +\int_{\sigma-r}^{\sigma} d \alpha\left(\int_{\sigma}^{t} Y(s, t) \eta(s, \alpha-s) d s\right) x(\alpha)+ \\
& +\int_{\sigma}^{t} d_{\alpha}\left(Y(\alpha, t)+\int_{\alpha}^{t} Y(s, t) \eta(s, \alpha-s) d s\right) x(\alpha)
\end{aligned}
$$

Usando (1.15) na quarta parcela, obtemos: 


$$
\begin{aligned}
X(t) & =Y(\sigma, t) X(\sigma)+\int_{\sigma}^{t} Y(s, t) h(s) d s+ \\
& +\int_{\sigma-r}^{\sigma} d \alpha\left(\int_{\sigma}^{t} Y(s, t) \eta(s, \alpha-s) d s\right) x(\alpha)
\end{aligned}
$$

provando-se assim (1.14).

$$
\text { Para o valor inicial }(\sigma, 0) \in R \times C \text {, temos por.(1.14) }
$$

que

$$
X(t)=\int_{\sigma}^{t} Y(s, t) h(s) d s \quad \text { para } \quad t \geq \sigma
$$

e pelo Teorema 1.12 temos

$$
\begin{aligned}
& X(t)=\int_{\sigma}^{t} U(t, s) h(s) d s \text { para } t \geq \sigma . \\
& \text { Portanto, } U(t, s)=Y(s, t) \text { q.s. em s. }
\end{aligned}
$$

\section{Comentārios Bibliogrāficos}

os fatos contidos nesta secção estão contidos em Hale, J.K. [7; secção 6.1-3]. Entretanto, a prova do Teorema 1.14 , aqui apresentada, é uma adaptação da de Banks, H.T. [1], essencialmente distinta da prova por Hale, J.K.

1.3. A Equação Diferencial Funcional Linear Autônoma com Retardamento

$$
\text { Consideremos a equação linear autônoma }
$$

$$
\text { (1.19) } \quad \dot{x}(t)=L\left(x_{t}\right)
$$

onde $L: C \rightarrow R^{n}$ é linear e continua.

Logo pelo Teorema 0.1 , existe uma matriz função $n(\theta)$, $\mathfrak{n} \times \mathfrak{n}$, de variação limitada em $[-r, 0]$ tal que

$$
L(\phi)=\int_{-r}^{0} \operatorname{dn}(\theta) \phi(\theta) \text {, para } \phi \in C .
$$


Veremos que as soluções da equação (1.19), sobre certo subespaço de $C$, tem características parecidas com as soluções de uma equação diferencial ordinäria linear com coeficientes constantes.

Para observarmos ofato acima, investigaremos as propriedades abstratas da aplicação solução associada a equação $(1.19)$. Se $\phi \in C$ e $x(\phi)$ ë a única solução da equação passando por $(0, \phi)$ a aplicação solução, $T(t): C \rightarrow C$ é definida por

(1.20) $T(t) \phi=x_{t}(\phi)$, para $t \geq 0$.

Com isto temos o:

\section{Lema 1.15}

o operador $T(t), \quad t \geq 0$ definido acima satisfaz as seguintes relações:

(i) $\{\mathrm{T}(\mathrm{t}), t \geq 0\}$ é um semigrupo de operadores lineares, isto $\vec{e}, T(0)=I$ e $T(t) T(s)=T(t+s)$ para $t, s \geq 0$.

(ii) $T(t)$ é limitada para cada $t \geq 0$ e $T(t)$ ë fortemente continua em $[0, \infty)$, isto é,

$\lim |T(t) \phi-T(s) \phi|=0$, para $t \geq 0$ e $\phi \in C$. $s \rightarrow t$

(iii) $T(t)$ é completamente contínua para $t \geq r$, isto $\vec{e}$, $T(t)$ é continua e leva conjunto limitado e fechado em conjunto relativamente compacto.

\section{Demonstração}

De (i)

Temos que $T(t): C+C$ dada por $T(t) \phi=x_{t}(\phi) \vec{e}$ um 
operador linear em vista da linearidade da equação (1.19) e pela unicidade temos que $x_{t+s}(\phi)=x_{t}\left(x_{s}(\phi)\right)$, portanto $T(t+s) \phi=$ $=T(t) T(s) \phi$, para $t, s \geq 0$ e $T(0) \phi=\phi$.

\section{De (ii)}

$T(t)$ é limitada para cada $t \geq 0$, pois como $L(t)$ ë linear e continua em $C$, segue que existe $l \geq 0$ tal que

$$
|L(\phi)| \leq \ell|\phi|
$$

para toda $\phi \in C$ e por (1.2) temos que

$T(t) \phi(\theta)=\phi(0)+\int_{0}^{t+\theta} L(T(s)) d s$, para $t+\theta \geq 0$ e $-r \leq \theta \leq 0$.

Logo,

$|\mathrm{T}(\mathrm{t}) \phi(\theta)| \leq|\phi(0)|+\int_{0}^{t+\theta}|\mathrm{L}(\mathrm{T}(\mathrm{s}))| \mathrm{ds}$.

Concluindo-se que,

$|T(t) \phi| \leq|\phi|+\int_{0}^{t} \ell|T(s) \phi| d s \quad$ para $\quad t \geq 0$.

Usando a Desigualdade de Gronwa11, segue que

$|\mathrm{T}(\mathrm{t}) \phi| \leq|\phi| \exp (\ell t)$.

Portanto, $T(t)$ é limitada para cada $t \geq 0$.

Mostremos que $T(t)$, é fortemente continua em $[0, \infty)$.

Para isto é suficiente mostrar que,

$$
\lim _{t \rightarrow 0^{+}}|T(t) \phi-\phi|=0
$$

Como,

$$
T(t) \phi(\theta)=\phi(0)+\int_{0}^{t+\theta} L(T(s)) d s \text { para } t+\theta \geq 0
$$

e 


$$
T(t) \phi(\theta)=\phi(t+\theta) \text { para }-r \leq t+\theta \leq 0
$$

segue o limite acima.

\section{$\underline{\text { De }(i i i)}$}

$T(t)$ é contínua para $t \geq 0$ e leva conjunto limitado e fechado em conjunto relativamente compacto para $t \geq r$, pois se $S=\{\phi \in C:|\phi| \leq R\}$ que $\bar{e}$ limitado e fechado, então:

$T(t) S \quad e ́$ uniformemente limitado pois,

$|\mathrm{T}(t) \phi| \leq|\phi| \exp (\ell t) \leq R \exp (\ell t)$ e alëm disso $|\dot{\psi}|=|\mathrm{L}(\psi)| \leq$ $\leq \ell|\psi| \leq \ell R \exp (\ell t)$, implicando que $T(t) S$ é equicontínuo, 10 go pelo Teorema de Ascoli-Arzela segue que $T(t) S$ é relativamen te compacto.

\section{Observações}

(1) As propriedades (i) e (ii) do Lema 1.5 nos diz que a família $T(t), \quad t \geq 0$ è um semigrupo de operadores $1 \underline{i}$ neares limitados fortemente continuos, isto $\bar{e}$, um comigrupo.

(2) A Desigualdade de Gronwall pode ser encontrada em [5, pag. 36] e o Teorema de Ascoli-Arze1a em [14, pag. 276].

\section{Definição 1.16}

Se $\{T(t), \quad t \geq 0\} \quad \overline{~ u m ~ s e m i g r u p o ~ d e ~ o p e r a d o r e s ~ l i-~}$ neares limitados fortemente continuos, definimos o gerador infi nitesimal, A, de $\{\mathrm{T}(\mathrm{t}), \mathrm{t} \geq 0\}$ como sendo

(1.21) $\quad \mathrm{A} \phi=\lim _{t \rightarrow 0^{+}} t^{-1}(\mathrm{~T}(t) \phi-\phi)$

para $\phi \in C$, quando este limite existe. 
o domínio de A será indicado por $D(A) \subset C$ e a ima gem de $A$ por $R(A) \subset C$.

Com isto temos o:

Lema 1.17

o gerador infinitesimal, A, do $C_{0}$-semigrupo. $T(t)$, $t \geq 0$, definido pela equação (1.19) pode ser dado por

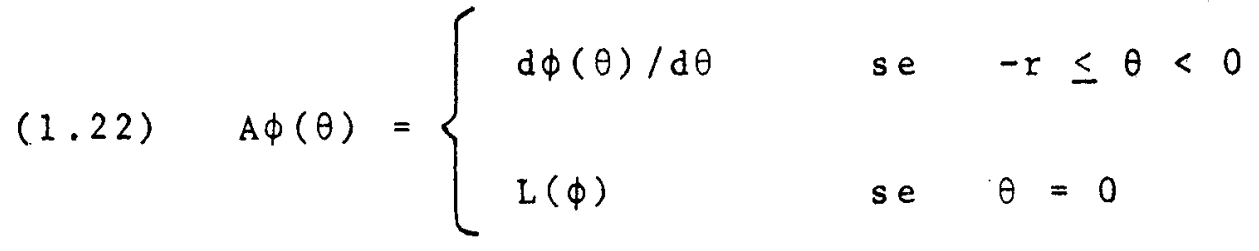

Além disso, A é um operador fechado, com $D(A)$ denso em C

e para $\phi \in D(A)$ temos

$(1.23)$

$$
\begin{aligned}
& \frac{d}{d t} T(t) \phi=T(t)(A \phi)=A(T(t) \phi) \\
& T(t) \phi-\phi=\int_{0}^{t} T(u)(A \phi) d u
\end{aligned}
$$

\section{Demonstração}

$$
\begin{aligned}
& \text { Para }-r \leq \theta<0,(1.21) \text {, implica que } \\
& \begin{aligned}
A \phi(\theta) & =\lim _{t \rightarrow 0^{+}} t^{-1}[\mathrm{~T}(t) \phi(\theta)-\phi(\theta)]= \\
& =\lim _{t \rightarrow 0^{+}} t^{-1}[\phi(t+\theta)-\phi(\theta)]=\frac{d^{+}}{d \theta} \phi(\theta)
\end{aligned}
\end{aligned}
$$

onde $\frac{\mathrm{d}^{+}}{\mathrm{d} \theta} \phi(\theta)$ indica a derivada a direita de $\phi$ em $\theta$.

$$
\begin{aligned}
& \text { Para } \theta=0, \text { temos } \\
& \begin{aligned}
A \phi(0) & =\lim _{t \rightarrow 0^{+}} t^{-1}[T(t) \phi(0)-\phi(0)]= \\
& =\lim _{t \rightarrow 0^{+}} t^{-1} \int_{0}^{t} L(T(s) \phi) d s=L(T(0) \phi)=L(\phi) .
\end{aligned}
\end{aligned}
$$


Como $R(A) \subset C$, segue de $(1.22)$ que $\phi \in D(A)$ se e somente se $\phi$ é continua, $\frac{d}{d \theta} \phi(\theta)$ é continua e $\dot{\phi}(0)=L(\phi)$. Logo segue (1.22).

$$
\begin{aligned}
& \text { Se } \phi \in O(A) \quad \text { então } \\
& \begin{aligned}
\frac{d}{d t} T(t) \phi(\theta)= & \lim _{s \rightarrow 0} \frac{1}{s}[T(t+s) \phi(\theta)-T(t) \phi(\theta)]= \\
= & \lim _{s \rightarrow 0} \frac{1}{s}[T(t) T(s) \phi(\theta)-T(t) \phi(\theta)]= \\
= & \left.T(t) \underset{s \rightarrow 0}{\left[1 i m \frac{1}{s}\right.}(T(s) \phi(\theta)-\phi(\theta))\right]=T(t) A \phi(\theta)
\end{aligned}
\end{aligned}
$$

e

$$
\begin{aligned}
\frac{d}{d t} T(t) \phi(\theta) & =\lim _{s \rightarrow 0} \frac{1}{s}[T(s) T(t) \phi(\theta)-T(t) \phi(\theta)]= \\
& =\operatorname{AT}(t) \phi(\theta)
\end{aligned}
$$

Logo segue $(1.23)$.

Como $\frac{d}{d t} T(t) \phi(\theta)=T(t) A \phi(\theta)$ então

$T(t) \phi(\theta)-\phi(\theta)=\int_{0}^{t} T(u) A \phi(\theta) d u$, isto $\bar{e}, T(t) \phi-\phi=\int_{0}^{t} T(u)(A \phi) d u$.

Mostremos que $D(A)$ é denso em $C$.

Paracada $\phi \in C$ e $t, s>0$, definimos

$A_{s}=\frac{1}{s}(T(s)-I)$, então:

$$
\begin{aligned}
& A_{s} \int_{0}^{t} T(u) \phi d u=\frac{1}{s} \int_{0}^{t}(T(s)-I) T(u) \phi d u= \\
= & \frac{1}{s} \int_{s}^{t+s} T(u) \phi d u-\frac{1}{s} \int_{0}^{t} T(u) \phi d u=\frac{1}{s} \int_{0}^{s} T(u)(T(t)-I) \phi d u .
\end{aligned}
$$

Logo,

$$
A_{S} \int_{0}^{t} T(u) \phi d u=\frac{1}{s} \int_{0}^{S} T(u)(T(t)-I) \phi d u .
$$


Fazendo $s \rightarrow 0^{+}$, temos que o lado direito da equação acima tende a $(T(t)-I) \phi$ em norma.

$$
\begin{aligned}
& \text { Logo, } \int_{0}^{t} T(u) \phi d u \in D(A) \text {. Usando o fato que } \\
& \lim _{t \rightarrow 0}+\frac{1}{t} \int_{0}^{t} T(u) \phi d u=\phi,
\end{aligned}
$$

segue que $O(A)$ é denso em $C$.

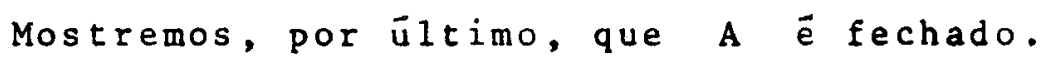

Seja $\left(\phi_{n}\right)$ uma seqüência em $D(A)$ convergindo para $\phi_{0} \operatorname{com} A \phi_{n}$ convergindo para $\psi_{0}$. Devemos mostrar que $\phi_{0} \in D(A)$ e $A \phi_{0}=\psi_{0}$.

Temos que

$$
\left|T(u)\left(A \phi_{n}\right)-T(u) \psi_{0}\right| \leq\left|A \phi_{n}-\psi_{0}\right| \exp (\ell u)
$$

e portanto converge uniformemente para zero com respeito a $u \in[0, t]$.

$$
\begin{aligned}
& \text { Para cada } n \text { temos: } \\
& T(t) \phi_{n}-\phi_{n}=\int_{0}^{t} T(u) A \phi_{n} d u
\end{aligned}
$$

e fazendo $n \rightarrow \infty$ em norma, obtemos

$$
\begin{aligned}
& T(t) \phi_{0}-\phi_{0}=\int_{0}^{t} T(u) \psi_{0} d u . \\
& \text { Logo } A_{s} \phi_{0}=\frac{1}{s} \int_{0}^{s} T(u) \psi_{0} d u \rightarrow \psi_{0}, \text { em norma, quando } s \rightarrow 0^{+},
\end{aligned}
$$

portanto, $\phi_{0} \in D(A)$ e $A \phi_{0}=\psi_{0}$.

\section{Observações}

(1) A demonstração do Lema 1.17 é uma adaptação da de Butzer, P.L. e Berens, H. em [3, pag. 9]. 
(2) Os fatos demonstrados no Lema 1.17 são fatos gerais da teoria de semigrupos de operadores lineares 1 imitados, como referência citamos ... Pazy, A. [13; Cap. 1]:

Para continuarmos, precisaremos de algumas definições.

\section{Definições 1.18}

Seja $B$ um espaço de Banach e $A: B+B$ um operador linear. Definimos o conjunto resolvente de $\mathrm{A}$, denotado por $\rho(A), \quad$ como:

$$
\begin{gathered}
\rho(A)=\left\{\lambda \in C \text { : existe }(\lambda-A)^{-1} \text { e é limitada num subconjunto den } .\right. \\
\text { so de } B\} .
\end{gathered}
$$

o complementar de $\rho(A)$ em $C$ será denominado espectro de A, denotado por $\sigma(\mathrm{A})$.

o.espectro de $A, \sigma(A)$, pode conter três tipos dife rentes de elementos, que são:

os $\lambda \in \sigma(A)$ tal que existe $(\lambda-A)^{-1}$, mas seu dominio não é denso em B, denominado espectro residual de $A$, de notado por $R \sigma(A)$; os $\lambda \in \sigma(A)$ tal que existe $(\lambda-A)^{-1}$ com domínio denso em $B$, mas não é limitada, denominado espectro continuo de $A$, denotado por $C \sigma(A)$; es $\lambda \in \sigma(A)$ tal que não existe $(\lambda-A)^{-1}$, denominado espectro pontual de $A$, deno tado por Po(A).

o espaco nulo ou núcleo de $\mathrm{A}$, denotado por $N(\mathrm{~A}), \overline{\mathrm{e}}$ o conjunto formado pelas $\phi \in B$ tal que $A \phi=0$. 
Se $\lambda \in, P \sigma(A)$ então $\lambda$ é dito autovalor dé $A$, e neste caso $N(\lambda-A) \neq\{0\}$ e $\phi \in N(\lambda-A), \phi \neq 0$ è dito autovetor dé A associado a $\lambda$.

Pära $\lambda \in \sigma(A)$, o auto-espaço generalizado por i, de notado por $M_{\lambda}(A)$, é definido como sendo o menor subespaço de B que contëm todos os elementos de $N(\lambda-A)^{k}, k=1,2, \ldots$

Nosso objetivo é determinar a natureza do $\sigma(T(t) ;$ e $\sigma(A)$ para os operadores dados pelas equações (1.20) e (1.22) e analisar em que sentido o operador $T(t)$ é aproximado por $e^{A t}$, contanto que este ültimo faça sentido. Para o caso em que a equa ção (1.19) è uma equação diferencial ordinäria, isto é, $r=0$, então $L(\phi)=A \phi(0)$, onde $A$ é uma matriz constante. Alëm disso, o gerador infinitesimal de $T(t) \quad \bar{a}$ e temos precisamente, $T(t)=e^{A t}$.

Como, em geral, $T(t)$ não é conhecido, nös esperamos poder discutir propriedades de $T(t)$ usando o gerador infinite simal A, dado por (1.22).

os resultados abaixo nos darão algumas propriedades de A.

\section{$\underline{\text { Lema } 1.19}$}

Se A é dado pela equação (1.22) então $\sigma(A)=P \sigma(A)$ e $\lambda \in \sigma(A)$ se e somente se $\lambda$ satisfaz a equação caracterís tica

(1.23) $\operatorname{det} \Delta(\lambda)=0$

onde $\Delta(\lambda)=\lambda I-\int_{-r}^{0} d n(\theta) e^{\lambda \theta}$, onde $I \quad$ e a matriz identidade. Além disso, as raízes da equação (1.23) têm parte real 
Iimitada superiormente; para cada $\lambda \in \sigma(A) \operatorname{dim} M_{\lambda}(A)<\infty \quad e$ existe $k \in N$ tal que $M_{\lambda}(A)=N(\lambda-A)^{k}$ e $C=M_{\lambda}(A) \oplus R(\lambda-A)^{k}$.

\section{Demonstração}

Para mostrar que $\sigma(A)=P \sigma(A)$, mostraremos que $O(A)$ $\vec{e}$ formado por todos os $\lambda \in C$ exceto os que satisfazem (1.23) e então mostraremos que se $\lambda$ satisfaz (1.23) então $\lambda \in \operatorname{Po}(\dot{A})$. Temos que: $\lambda \in \rho(A)$ se e somente se a equação

$(1.24) \quad(A-\lambda I) \phi=\psi$

tem uma solução $\phi \in D(A)$ para cada. $\psi$ num subconjunto denso de $C$ e $\phi$ depende continuamente de $\psi$.

Como $\phi \in D(A)$ segue que $\phi$ é continuamente diferenciável e $\dot{\phi}(\theta)=A \phi(\theta), \quad-r \leq \theta \leq 0,10 g \circ$ se $\phi$ è uma solução de $(1.24)$ ela satisfaz:

$$
\begin{aligned}
& \dot{\phi}(\theta)-\lambda \phi(\theta)=\psi(\theta), \text { para }-r \leq \theta \leq 0 . \\
& \text { Logo, } \dot{\phi}(\theta)=\lambda \phi(\theta)+\psi(\theta),-r \leq \theta \leq 0, \text { cuja soluçã , }
\end{aligned}
$$
dada pela fórmula da variação das constantes é (1.25) $\phi(\theta)=e^{\lambda \theta} b+\int_{0}^{\theta} e^{\lambda(\theta-\xi)} \psi(\xi) d \xi, \quad-r \leq \theta \leq 0$, com $b=\phi(0)$. Mas,

$$
\begin{aligned}
& \lambda \phi(0)+\psi(0)=\dot{\phi}(0)=L(\phi)=\int_{-r}^{0} d r(\theta) \phi(\theta)= \\
& =\int_{-r}^{0} d \eta(\theta)\left[e^{\lambda \theta} b+\int_{0}^{\theta} e^{\lambda(\theta-\xi)} \psi(\xi) d \xi\right]
\end{aligned}
$$

Donde segue

$$
\left[\lambda I-\int_{-r}^{0} d \eta(\theta) e^{\lambda \theta}\right] b=-\psi(0)+\int_{-r}^{0} \operatorname{dn}(\theta)\left[\int_{0}^{\theta} e^{\lambda(\theta-\xi)} \psi(\xi) d \xi\right]
$$


is to $\bar{e}$,

(1.26) $\Delta(\lambda) b=-\psi(0)+\int_{-r}^{0} \mathrm{~d} \eta(\theta)\left[\int_{0}^{\theta} e^{\lambda(\theta-\xi)} \psi(\xi) \mathrm{d} \xi\right] \mathrm{de} f \underline{\underline{e}}-(\alpha, \psi)$ onde $\alpha$ é definida por $\alpha(s)=e^{-\lambda s} I, \quad 0 \leq s \leq r$

e

(1.27) $(\alpha, \psi)=\alpha(0) \psi(0)-\int_{-r}^{0} \mathrm{dn}(\theta)\left[\int_{0}^{\theta} \alpha(\xi-\theta) \psi(\xi) \mathrm{d} \xi\right]$.

De $(1.25)$ e (1.26) segue que (1.24) tem uma solução $\phi \in D(A)$ para $\psi \in C$ se e somente se det $\Delta(\lambda) \neq 0$, pois $(\alpha, \cdot)$ cobre o $R^{n}$. Portanto, $\rho(A)=\{\lambda \in C: \operatorname{det} \Delta(\lambda) \neq 0\}$.

Se $\operatorname{det} \Delta(\lambda)=0$ então de (1.25) e (1.26), existe uma solução, não nula, de (1.24) para $\psi \equiv 0,10 g o$ não existe $(A-\lambda I)^{-1}$ e portanto $\lambda \in P \sigma(A)$, concluindo-se que $P \sigma(A)=\sigma(A)$.

A função característica det $\Delta(\lambda)$ é uma função inteira em $\lambda$ e portanto os zeros tem ordem finita, logo o operador $(A-\lambda I)^{-1}$ tem um polo de ordem $k$ em $\lambda$ se $\operatorname{det} \Delta(\lambda)=0$. Como A é fechado, usando um resultado de [14, pag. 306] segue que $N(A-\lambda I)^{k}=M_{\lambda}(A)$ e $C=M_{\lambda}(A) \oplus R(A-\lambda I)^{k}$.

Para mostrar que $\operatorname{dim} M_{\lambda}(A)$ é finita usamos um resulta do de $[9, \mathrm{pag}, 187]$.

Por ültimo, como a equação característica é um polinômio de grau n em $\lambda$, com coeficiente líder um e termo de me nor grau tem coeficiente que depende de $\lambda$ atravês de integrais sobre $[-r, 0]$ de termos da forma $e^{\lambda \theta}$, segue que existe $\gamma>0$, tal que, não existem raízes de (1.23) $\operatorname{com} \operatorname{Re} \lambda>\gamma$, concluindo assim a demonstração do Lema.

Do Lema 1.19 , sabemos que se $\lambda \in \sigma(A)$ entäo $\operatorname{dimM}_{\lambda}(A)<\infty$ e existe $k \in N$ tal que $M_{\lambda}(A)=N(A-\lambda I)^{k}$. o subespaço $M_{\lambda}(A)$ 
é A-invariante, isto é, $\quad \operatorname{AM}_{\lambda}(A) \subset M_{\lambda}(A)$.

Seja $\left\{\phi_{1}^{\lambda}, \ldots, \phi_{d}^{\lambda}\right\}$ uma base para $M_{\lambda}(A)$ e tomemos $\Phi_{\lambda}=\left(\phi_{1}^{\lambda}, \ldots, \phi_{d}^{\lambda}\right)$; como $M_{\lambda}(A)$ e A-invariante, existe uma matriz, ${ }_{\lambda}$, d $x$ d tal que ${ }^{A} \Phi_{\lambda}=\Phi_{\lambda}{ }_{\lambda} \cdot 0$ autovalor de ${ }^{B}{ }_{\lambda}$ é $\lambda$.

Da definição de ${ }_{B}$ em $(1.22)$, a relação ${ }^{A \Phi} \Phi_{\lambda}=\Phi_{\lambda}{ }^{B}{ }_{\lambda}$, implica que $\Phi_{\lambda}(\theta)=\Phi_{B}(0) e^{B} \lambda^{\theta}, \quad-r \leq \theta \leq 0$. Logo pelo Lema 1.17 obtemos $T(t) \Phi_{\lambda}=\Phi_{\lambda} e^{B} \lambda^{t}$, para $t \geq 0$.

Portanto, no auto-espaço generalizado por um autovalor $\lambda \in \sigma(A)$, a equação $(1,19)$ tem a mesma estrutura de uma equação diferencial ordinária linear com coeficientes constantes. Além disso, segue também do Lema 1.17 que $R(A-\lambda I)^{k}$ é $T(t)$-invarian te.

Pela aplicação repetida do processo acima nós obtemos:

\section{Teorema 1.20}

Suponhamos que $\Lambda=\left\{\lambda_{1}, \ldots, \lambda_{p}\right\}$ e um conjunto finito de autovalores da equação $(1.19)$, que $\Phi_{\Lambda}=\left(\Phi_{\lambda_{1}}, \ldots, \Phi_{\lambda_{p}}\right)$, on de $\Phi_{\lambda_{j}}$ é uma base para o auto-espaço generalizado por $\lambda_{j}$ e ${ }^{B} \Lambda=\operatorname{diag}\left(B_{\lambda_{1}}, \ldots, B_{\lambda_{p}}\right)$, onde $B_{\lambda_{j}} \vec{e}$ a matriz definida por ${ }^{A} \Phi_{\lambda_{j}}=\Phi_{\lambda}{ }_{j}^{B} \lambda_{j}$, com $j=1, \ldots, p$. Então o único autovalor de ${ }^{B} \lambda_{j} \quad \bar{e} \lambda_{j}$ e para cada vetor $a$, de mesma dimensão de $\Phi_{\Lambda}$, a solução $T(t) \Phi_{\Lambda}$, com condição inicial $\Phi_{\Lambda}$ a em $t=0$ pode ser definida em $R$ pela relação

(1.28) $\quad T(t) \Phi_{\Lambda} a^{a}=\Phi_{\Lambda} e^{B} \Lambda^{t} a$

$\operatorname{com} \Phi_{\Lambda}(\theta)=\Phi_{\Lambda}(0) e^{B} \Lambda^{\theta},-r \leq \theta \leq 0$. 
Além disso, existe um subespaço $Q_{\Lambda} \subset C$ tal que $T(t) Q_{\Lambda} \subset Q_{\Lambda}$, para $t \geq 0$ e $C=P_{\Lambda} \oplus Q_{\Lambda}$ onde $P_{\Lambda}=\left\{\phi \in C: \phi=\Phi_{\Lambda} b\right.$ para algum vetor b\}.

Daremos, a seguir, um resultado sobre o comportamento dos autovalores simples de uma equação linear autônoma que depende de um parâmetro real.

\section{Definição 1.21}

Dizemos que $\lambda$ è um autovalor simples de uma EDFR(L), onde $L$ é linear, se $\lambda$ é uma raiz simples da equação caracteristica associada a $\operatorname{EDFR}(L)$.

\section{Lema 1.22}

Suponhamos que $\{L(\alpha): \alpha \in R\}$ é uma família de operadores lineares limitados de $C$ em $R^{n}$, continuos juntamente com suas primeiras derivadas em $\alpha$. Se $\lambda_{0}$ é um autovalor sim ples da EDFR $(L(0))$, então existe $\alpha_{0}>0$ e um autovalor simples, $\lambda(\alpha)$, da EDFR(L( $\alpha)$ ) que é continuo juntamente com sua primeira derivada para $|\alpha|<\alpha_{0}$ e $\lambda(0)=\lambda_{0}$.

\section{Demonstração}

Consideremos a equação

(1.29) $\Delta(\alpha, \lambda) b=0$,

onde $\Delta(\alpha, \lambda)=\lambda(\alpha) I-L(\alpha)\left(e^{\lambda(\alpha)} \cdot I\right)$.

Como $\lambda_{0}$ é um autovalor simples da EDFR(L(0)), então $\operatorname{dim} N\left(\Delta\left(0, \lambda_{0}\right)\right)=1$ e $C^{\mathfrak{n}}=N\left(\Delta\left(0, \lambda_{0}\right)\right) \oplus R\left(\Delta\left(0, \lambda_{0}\right)\right)$. Sejam $p$ e (I-p) as projeções definidas por esta decomposição.

Reescrevendo ( 1.29 ) temos, 
$\Delta(a, \lambda) b=0=\Delta\left(0, \lambda_{0}\right) b-\Delta\left(0, \lambda_{0}\right) b$

Logo,

(1.30) $\Delta\left(0, \lambda_{0}\right) b=\left[\Delta\left(0, \lambda_{0}\right)-\Delta(\alpha, \lambda)\right] b$.

Fizemos $b_{0} \in N\left(\Delta\left(0, \lambda_{0}\right)\right)$. Se $b=b_{0}+d, \quad \operatorname{com} d \in(I-p) C^{n}$ então a equação (1.30) é equivalente às equações,

$(1.31 . a) \Delta\left(0, \lambda_{0}\right) d=(I-p)\left[\Delta\left(0, \lambda_{0}\right)-\Delta(\alpha, \lambda)\right]\left(b_{0}+d\right)$

$(1.31 . b) \quad p\left[\Delta\left(0, \lambda_{0}\right)-\Delta(\alpha, \lambda)\right]\left(b_{0}+d\right)=0$

Como $\Delta\left(0, \lambda_{0}\right)$ e um isomortismo de $(I-p) C^{n}$ em $(I-p) C^{n}$ e é continua juntamente com sua primeira derivada, do teorema da função implícita segue que existe $\delta>0$ e uma ünica solução $\mathrm{d}^{*}=\mathrm{d} *\left(\alpha, \lambda, \mathrm{b}_{0}\right)$ de $(1.31 . \mathrm{a})$ para $\left|\lambda-\lambda_{0}\right|<\delta,|\alpha|<\delta$, conti nuamente diferenciável em $a, \lambda, b_{0}$ e $d *\left(0, \lambda_{0}, b_{0}\right)=0$. Podemos escrever, $d *\left(\alpha, \lambda, b_{0}\right)=D *(\alpha, \lambda) b_{0}$, onde $D *(\alpha, \lambda)$ é uma ma triz $n \times n$. Logo se b satisfaz $\Delta(\alpha, \lambda) b=0, \operatorname{com} b=b_{0}$ para $\alpha=\alpha_{0}$ e $\lambda=\lambda_{0}$, então $b=b_{0}+D *(\alpha, \lambda) b_{0}$, para $|\alpha|<\delta$ e $\left|\lambda-\lambda_{0}\right|<\delta$ e

(1.32) $f(\alpha, \lambda) b_{0} d \stackrel{d e f}{=} P\left[\Delta\left(0, \lambda_{0}\right)-\Delta(\alpha, \lambda)\right][I+D *(\alpha, \lambda)] b_{0}=0$.

Logo, a existência de um autovalor $\lambda$ pröximo a $\lambda_{0}$, para a próximo de zero é equivalente a existir uma solução de (1.32).

Como,

$\Delta\left(0, \lambda_{0}\right) \frac{\partial D *\left(0, \lambda_{0}\right)}{\partial \lambda}=-(I-P) \frac{\partial \Delta\left(0, \lambda_{0}\right)}{\partial \lambda}$

então, 


$$
\frac{\partial f\left(0, \lambda_{0}\right)}{\partial \lambda} b_{0}=-p \frac{\partial \Delta\left(0, \lambda_{0}\right)}{\partial \lambda} b_{0} \cdot
$$

Escolhendo-se uma base tal que $\Delta\left(0, \lambda_{0}\right)=\operatorname{diag}(0, B)$, onde $B$ é uma matriz $(n-1) \times(n-1)$, não singular, podemos to mar $b_{0}=(1,0, \ldots, 0)$ e a projeção $p$ pode ser identificada por $(1,0, \ldots, 0)$. Então $p \Delta(0, \lambda) b_{0}=a(\lambda)$ onde $a(\lambda)$ è o ele mento da primeira linha e da primeira coluna de $\Delta(0, \lambda)$. Com is to temos que

$$
p\left[\frac{\partial \Delta\left(0, \lambda_{0}\right)}{\partial \lambda}\right] b_{0}=(\operatorname{det} B)^{-1} \frac{\partial \operatorname{det} \Delta\left(0, \lambda_{0}\right)}{\partial \lambda}
$$

Logo,

$$
\frac{\partial f\left(0, \lambda_{0}\right)}{\partial \lambda} b_{0}=-(\operatorname{det} B)^{-1} \frac{\partial \operatorname{det} \Delta\left(0, \lambda_{0}\right)}{\partial \lambda} \neq 0
$$

pois $\lambda_{0} \vec{e}$ autovalor simples da $\operatorname{EDFR}(L(0))$.

$$
\text { Portanto, } \frac{\partial f\left(0, \lambda_{0}\right)}{\partial \lambda} b_{0} \neq 0 \text { e por }(1.32) \quad f\left(0, \lambda_{0}\right)=0,
$$

logo pelo teorema da função implicita segue que existe $\bar{S}>0$ e uma função continuamenfe diferenciável, $\lambda(\alpha), \operatorname{com} \lambda(0)=\lambda_{0}$ tal que $f(\alpha, \lambda(\alpha))=0$ para $|\alpha|<\delta$. Para completaro resul tado basta tomar $\alpha_{0}=\min \{\delta, \bar{\delta}\}$.

Os resultados que seguem nos darão uma decomposição de C pela equação adjunta formal associada à equação (1.19), isto é, ’a equação linear autônoma.

Para isto, seja $C^{*}=C\left([0, r], R^{n *}\right)$ e para $\alpha \in C^{*}$ e $\phi \in C$ definamos

(1.33) $(\alpha, \phi)=\alpha(0) \phi(0)-\int_{-\zeta}^{0} \mathrm{~d} \eta(\theta)\left[\int_{0}^{\theta} \alpha(\xi-\theta) \phi(\theta) \mathrm{d} \xi\right]$ 
que è uma forma bilinear.

$$
\text { Definamos } A^{*}: D\left(A^{*}\right)^{*} \rightarrow C^{*} \text { tal que se } \alpha \in D\left(A^{*}\right)
$$

(1.34) $\quad\left(A *_{\alpha}\right)(s)= \begin{cases}-\frac{d \alpha(s)}{d s} & \text { se } 0<s \leq r \\ \int_{-r}^{0} \operatorname{dn}(\theta) \alpha(-\theta) & \text { se } s=0\end{cases}$

onde $D\left(A^{*}\right)$, consiste de todas as $\alpha \in C^{*}$ que tem primeira derivada continua eque satisfazem

$$
-\frac{d \alpha(0)}{d s}=\int_{-r}^{0} \alpha \operatorname{dn}(\theta) \alpha(-\theta) .
$$

observemos que $D\left(A^{*}\right)$ é denso em $C^{*}$ e que se $\alpha \in D\left(A^{*}\right)$

e $\phi \in D(A)$ então

$(1.35) \quad(\alpha, A \phi)=(A * \alpha, \phi)$.

\section{Definição 1.23}

Diremos que $A^{*}$, dada pela expressão (1.34) è a adjun ta formal de A relativo a (1.35).

A equação adjunta formal, para a equação (1.19) $\vec{e}$

$(1.36) \quad y \dot{y}(s)=-\int_{-r}^{0} \operatorname{dn}(\theta) y(s-\theta)$.

Defato, pois se $y(s)+\int_{s}^{\infty} y(\alpha) \eta(s-\alpha) d \alpha=$ cte, então $\dot{y}(s)=-\frac{d}{d s} \int_{s}^{\infty} y(\alpha) \eta(s-\alpha) d \alpha$.

Como, $\int_{s}^{n} y(\alpha) \eta(s-\alpha) d \alpha \rightarrow \int_{s}^{\infty} y(\alpha) \eta(s-\alpha) d \alpha$ quando $n \rightarrow \infty$, temos que 


$$
\begin{aligned}
& \frac{d}{d s} \int_{s}^{n} y(\alpha) \eta(s-\alpha) d \alpha=\int_{s}^{n} y(\alpha) \eta^{\prime}(s-\alpha) d \alpha= \\
& =-\int_{0}^{s-n} y(s-\tau) \eta^{\prime}(\tau) d \tau=\int_{s-n}^{0} d \eta(\tau) y(s-\tau) .
\end{aligned}
$$

Além disso, $\frac{d}{d s} \int_{s}^{n} y(\alpha) n(s-\alpha) d \alpha \rightarrow \int_{-\infty}^{0} d n(\theta)$ y $(s-\theta)$ uniformemente quando $n \rightarrow \infty$, pois

$$
\frac{d}{d s} \int_{s}^{n} y(\alpha) \eta(s-\alpha) d \alpha=\int_{s}^{n} y(\alpha) \eta^{\prime}(s-\alpha) d \alpha
$$

e a integral do segundo membro da igualdade é feito no interva$10[s, s+r]$, para $n \geq s+r$, pois se $n \geq \alpha \geq s+r$ então $s-\alpha \leq-r$ concluindo-se que $\eta(s-\alpha)=\eta(-r), \quad \log 0 \eta^{\prime}(s-\alpha)=0$ se $\alpha \geq s+r$. Logo quando $n \geq s+r$ temos que

$$
\frac{d}{d s} \int_{s}^{n} y(\alpha) \eta(s-\alpha) d \alpha=\int_{s}^{s+r} y(\alpha) \eta^{\prime}(s-\alpha) d \alpha .
$$

Portanto, $\quad \frac{d}{d s} \int_{s}^{n} y(\alpha) \eta(s-\alpha) d \alpha$ converge uniformemente $\operatorname{para} \int_{-\infty}^{s} \operatorname{dn}(\theta) y(s-\theta)$.

Se y(s) é uma solução da equação (1.36) em $(-\infty, r]$ tal que $y(\tau)=\alpha(\tau)$ para $0 \leq \tau \leq r$ e $\alpha \in C^{*}$, definimos, para $s \leq 0$ a aplicação, $T *(s): C * \rightarrow C *$ dada por $T *(s) \alpha=y s(\alpha)$, $\alpha \in C^{*}$, onde $y^{s}(\alpha)(\tau)=y(\tau+s)$ para $0 \leq \tau \leq r$. Neste caso $T *(s), \quad s \leq 0$, tem as mesmas propriedades do semigrupo associa do a equação $(1.19)$ e

$$
\frac{d}{d s} T *(s) \alpha=-A * T *(s) \alpha=-T *(s) A * \alpha
$$

para $\alpha \in D\left(A^{*}\right), \quad s \leq 0$. 
Se $x$ é uma solução da equação (1.19) e y é solução da equação ( 1.36$)$, um simples cālculo, nos mostra que $\left(y^{t}, x_{t}\right)=c t e$, $\forall t$.

Daremos a seguir um lema que nos dará informaçoes importantes com respeito a $\sigma\left(A^{*}\right)$.

\section{Lema 1.24}

$\lambda \in \sigma(A)$ se e somente se $\lambda \in \sigma\left(A^{*}\right) ; \sigma\left(A^{*}\right)=P \sigma\left(A^{*}\right)$ e para todo $\lambda \in \sigma\left(A^{*}\right)$ o auto-espaço generalizado por $\lambda, M_{\lambda}\left(A^{*}\right)$ tem dimensão finita.

\section{Demonstração}

A ültima parte do Lema é provada como no Lema 1.19 .

Temos que $\lambda \in \sigma\left(A^{*}\right)$ se e somente se $\alpha(s)=e^{-\lambda s} b$, com $0 \leq s \leq r$, onde $b \in R^{n \star}$, não nulo, satisfazendo

$$
b\left[\lambda I-\int_{-r}^{0} d n(\theta) e^{\lambda \theta}\right]=b \Delta(\lambda)=0,
$$

isto é, $\operatorname{det} \Delta(\lambda)=0$, ou ainda $\lambda \in \sigma(A)$.

o pröximo resultado: fornece uma relação entre $R(A-\lambda I)^{k}$ e $N(A-\lambda I)^{k}$, cuja demonstração pode ser encontrada em [7; pag. $175]$.

\section{Lema 1.25}

Uma condição neçessária e suficiente para a equação

$$
(A-\lambda I)^{k}=\psi, \psi \in C
$$

ter uma solução $\phi \in C$, isto é, $: \psi \in R(A-\lambda I)^{k}$ é que $(\alpha, \psi)=0$ para todo $a \in N(A *-\lambda I)^{k}$. 
Como conseqüência dos resultados anteriores temos:

Lema 1.26

Para $\lambda \in \sigma(A)$, seja $\Psi=\operatorname{col}\left(\psi_{1}, \ldots, \psi_{p}\right)$ e

$\Phi_{\lambda}=\left(\phi_{1}, \ldots, \phi_{p}\right)$ bases para $M_{\lambda}\left(A^{*}\right)$ e $M_{\lambda}(A)$ respectivamente e seja a matriz $\left(\Psi_{\lambda}, \Phi_{\lambda}\right)=\left(\left(\psi_{i}, \phi_{j}\right)\right), \quad i, j=1, \ldots, p$. Então $\left(\Psi_{\lambda}, \Phi_{\lambda}\right)$ è não singular e portanto pode ser tomada como a identidade. A decomposição de $C$ dada pelo Lema 1.19 pode ser escrita explicitamente como

$$
\begin{aligned}
& \phi=\phi^{P_{\lambda}}+\phi^{Q_{\lambda}} \text { onde } \quad \phi^{P_{\lambda}} \in \mathrm{P}_{\lambda} \text { e } \phi^{Q_{\lambda}} \in Q_{\lambda} \text {, } \\
& P_{\lambda}=M_{\lambda}(A)=\left\{\phi \in C: \phi=\Phi_{\lambda} b \text { para algum p-vetor b }\right\} \\
& P_{\lambda}=\left\{\phi \in C:\left(\Psi_{\lambda}, \phi\right)=0\right\} \\
& \phi^{P} \lambda=\phi_{\lambda} b \text { onde } b=\left(\Psi_{\lambda}, \phi\right)
\end{aligned}
$$

E interessante notar que se $\left(\Psi_{\lambda}, \Phi_{\lambda}\right)=I$ e $B_{\lambda}^{*}$ è uma matriz tal que $A_{\lambda}^{* \Psi}=B_{\lambda}^{* \Psi} \lambda$ então $B_{\lambda}^{*}=B_{\lambda}$.

Nós jā definimos o auto-espaço generalizado por um au tovalor para a equação (1.19), que é $M_{\lambda}(A)$, Daremos agora a definição de auto-espaço generalizado para um conjunto finito de autovalores para a equação (1.19). Se $\Lambda=\left\{\lambda_{1}, \ldots, \lambda_{p}\right\}$ é um conjunto finito de autovalores para a equação (1.19) então seja ${ }^{P} \Lambda$ a extensão linear de $M_{\lambda_{j}}(A), j=1, \ldots, p$, e nos referire mos a este subespaço como o auto-espago generalizado para a equasão (1.19) associado a $\Lambda$. Do mesmo modo obtemos $P_{\lambda}^{*}$ que serà o auto-espago generalizado para a equacão adjunta (1.36) associado a $\Lambda$. Se $\dot{\Phi}, \Psi$ são bases para $\mathrm{P}_{\Lambda}$ e $\mathrm{P}_{\Lambda}^{*}$, rës pectivamente, $\operatorname{com}(\Psi, \Phi)=I$ então 


$$
\begin{aligned}
& C=P_{\Lambda} \oplus Q_{\Lambda} \\
& P_{\Lambda}=\{\phi \in C: \phi=\phi b, \text { para algum vetor } b\} \\
& Q_{\Lambda}=\{\phi \in C:(\Psi, \phi)=0\} \\
& \phi={ }^{P} \Lambda+{ }^{P}{ }^{Q}
\end{aligned}
$$

onde $\quad \phi^{P_{\Lambda}}=\phi(\Psi, \phi)$.

Os resultados que seguem nos darão, de maneira explicita, a derivada do autovalor $\lambda(\alpha)$ dado no Lema 1.22. Para isto precisaremos do:

\section{$\underline{\text { Lema } 1.27}$}

$\operatorname{be}_{0}{ }^{\theta}, \quad-r \leq \theta \leq 0, \psi_{0}(s)=a e^{-\lambda_{0} s}, 0 \leq s \leq r$, são bases para $N(A-\lambda I)$ e $N\left(A^{\star}-\lambda I\right)$, respectivamente, então

$$
\left(\psi_{0}, \phi_{0}\right)=a \frac{\partial \Delta\left(\lambda_{0}\right)}{\partial \lambda} b \text {. }
$$

\section{Demonstração}

Da definição da forma bilinear temos

$$
\begin{aligned}
& \left(\psi_{0}, \phi_{0}\right)=\psi_{0}(0) \phi_{0}(0)-\int_{-r}^{0} \mathrm{dn}(\theta) \int_{0}^{\theta} \psi_{0}(\xi-\theta) \phi_{0}(\xi) d \xi= \\
= & a b-\int_{-r}^{0} d \eta(\theta) \int_{0}^{\theta} a e^{\lambda_{0} \theta} b d \xi=a b-a \int_{-r}^{0} d \eta(\theta) \theta^{\lambda_{0} \theta_{b}}= \\
= & a\left[I-\int_{-r}^{0} d \eta(\theta) \theta e^{\lambda_{0}{ }^{\theta}}\right] b=a \frac{\partial \Delta\left(\lambda_{0}\right)}{\partial \lambda} b
\end{aligned}
$$




\section{Lema 1.28}

Suponhamos que as condições do Lema 1.22 estão satisfei tas e sejam $\phi_{\alpha}(\theta)=b(\alpha) \mathrm{e}^{\lambda(\alpha) \theta},-r \leq \theta \leq 0$ e $\psi_{\alpha}(s)=a(\alpha) \mathrm{e}^{-\lambda(\alpha) s}$, para $0 \leq s \leq r$, bases para $N(A-\lambda(\alpha) I)$ e $N\left(A^{*}-\lambda(\alpha) I\right)$, respectivamente, para o autovalor simples $\lambda(\alpha)$ da EDFR(L( $\alpha)$ ).

se $\left(\psi_{\alpha}, \phi_{\alpha}\right)=1$ então

$\lambda^{\prime}(\alpha)=+a(\alpha) L^{\prime}(\alpha)\left(e^{\lambda(\alpha)} \cdot I\right) b(\alpha)$

para todo $\alpha \in R$, onde " " denota a derivada com relação a $\alpha$.

\section{Demonstração}

Da definição de $a(\alpha)$ e $b(\alpha)$, temos

$a(\alpha) \Delta(\alpha, \lambda(\alpha))=0 \quad$ e $\quad \Delta(\alpha, \lambda(\alpha)) b(\alpha)=0$

Logo, $a(\alpha) \Delta(\alpha, \lambda(\alpha)) b(\alpha)=0$, para todo $\alpha \in R$.

Derivando esta expressão com respeito a $\alpha$ e usando o

Lema 1.27 obtemos,

$$
\lambda^{\prime}(\alpha)=a(\alpha) \frac{\partial \Delta(\alpha, \lambda(\alpha))}{\partial \alpha} b(\alpha), \text { para todo } \alpha \in R
$$

Mas,

$$
\begin{gathered}
\frac{\partial \Delta(\alpha, \lambda(\alpha))}{\partial \alpha}=\lambda^{\prime}(\alpha)\left[I-L(\alpha)\left(e^{\lambda(\alpha)} \cdot I\right)\right]- \\
-L^{\prime}(\alpha)\left(e^{\lambda(\alpha)} \cdot I\right)=\lambda^{\prime}(\alpha) \frac{\partial \Delta(\alpha, \lambda(\alpha))}{\partial \lambda(\alpha)}-L^{\prime}(\alpha)\left(e^{\lambda(\alpha)} \cdot I\right)
\end{gathered}
$$

Portanto,

$$
\lambda^{\prime}(\alpha)=+a(\alpha) L^{\prime}(\alpha)\left(e^{\lambda(\alpha)} \cdot I\right) b(\alpha), \text { para } \alpha \in R
$$


Lema 1.29

Suponhamos que as hịpóteses do Lema 1.22 estão satisfeitas e que $\Lambda(\alpha)=\left\{\lambda_{1}(\alpha), \ldots, \lambda_{p}(\alpha)\right\}$ e um conjunto finito de autovalores simples da EDFR(L( $\alpha)) \operatorname{com} \lambda_{2 j-1}(\alpha)=\bar{\lambda}_{2 j}(\alpha)$, $j=1, \ldots, k$ e $\lambda_{j}(\alpha)$ è real para $j=2 k+1, \ldots, p$. Sejam $\Phi_{\alpha}$, $\Psi_{\alpha}$ bases reais para $M_{\Lambda(\alpha)}(A)$ e $M_{\Lambda(\alpha)}\left(A^{*}\right)$, respectivamente, $\operatorname{com}\left(\Psi_{\alpha}, \Phi_{\alpha}\right)=I$ e $B(\alpha)$ uma matriz tal que $A \Phi_{\alpha}=\Phi_{\alpha} B(\alpha)$. EI tão

$$
B^{\prime}(\alpha)=+\Psi_{\alpha}(0) L^{\prime}(\alpha)\left(\Phi_{\alpha}(0) e^{B(\alpha)} \cdot\right) \text {, para todo } \alpha \in R \text {. }
$$

A demonstração do Lema 1.29 , pode ser encontrada em $[7 ;$ pag. 179].

Para as aplicaçós da teoria de sistemas lineares autô nomos nós precisaremos ter uma estimativa para as soluções da e quação (1.19) no subespaço complementar de $P_{\Lambda}, Q_{\Lambda}$. Para isto temos o resultado a seguir, cuja prova pode ser encontrada em $[7 ;$ pag. 181$]$.

\section{Teorema 1.30}

Para todo $\beta \in R$, seja $\Lambda=\Lambda(\beta)=\{\lambda \in \sigma(A): \operatorname{Re} \lambda \geq \beta\}$ e suponhamos que $C$ é decomposto por $\Lambda \operatorname{como} C=P_{\Lambda} \oplus Q_{\Lambda}$. En tão existem constantes $K, \gamma \geqslant 0$ tal que

$$
\left\|T(t) \phi^{P} \Lambda\left|\leq K e^{(B+\gamma) t}\right|\right\|^{P} \Lambda|| \text { para } t \leq 0
$$

e

$$
\left\|\mathrm{T}(\mathrm{t}) \phi^{\mathrm{Q}} \Lambda\left|\leq \mathrm{Ke}^{(\beta-\gamma) t} \|^{\mathrm{P}} \Lambda \phi\right| \text { para } \mathrm{t} \geq 0 .\right.
$$




\subsection{A Equação Linear Autionoma Forçada}

Nesta secção, nós consideraremos propriedades da equação linear homogênea

(1.39) $\dot{x}(t)=L\left(x_{t}\right)=\int_{-r}^{0} d \eta(\theta) x(t+\theta)$

que assegurarão que a equação não homogênea

$(1.40) \quad \dot{x}(t)=L\left(x_{t}\right)+f(t)$

tenha, pelo menos, uma solução numa classe $B$ se $f \in B$. A classe $B$ pode ser o conjunto das funçóes definidas em $R, R^{+}$ ou $R^{-}$e tomando valores em $R^{n}$, continuas e limitadas, ou continuas e periódicas, como veremos a seguir.

para $\sigma \in R, j a ̄$ mostramos, pela fórmula da variação das constantes na secção 1.2 , que a solução $x$ da equação $(1.40)$ com condição inicial $x_{\sigma}$ em $\sigma$ pode ser escrita como

$(1.41) \quad x_{t}=T(t-\sigma) x_{\sigma}+\int_{\sigma}^{t} T(t-s) x_{0} f(s) d s, t \geq \sigma$.

$$
\begin{aligned}
& \text { Suponhamos que } \Lambda=\Lambda_{0} \cup \Lambda_{1} \text { onde, } \\
& \Lambda_{0}=\{\lambda \in \sigma(A): \operatorname{Re} \lambda=0\} \text { e } \Lambda_{1}=\{\lambda \in \sigma(A): \operatorname{Re} \lambda>0\},
\end{aligned}
$$

onde $A$ é o gerador infinitesimal gerado pela equação (1.39). Se $P_{0} e P_{1}$ são os auto-espaços generalizados da equação (1.39) associados $\operatorname{com} \Lambda_{0}$ e $\Lambda_{1}$, respectivamente, e $\quad C=P_{0}^{\oplus P}{ }_{1}^{\oplus Q}$ então a decomposição da solução

$$
x_{t}=x_{t}^{P_{0}}+x_{t}^{P_{1}}+x_{t}^{Q}
$$

torna a equação ( 1.41 ) equivalente às equaçöes: 
$(1.42 . a) x_{t}^{P_{0}}=T(t-\sigma) x_{t}^{P_{0}}+\int_{\sigma}^{t} T(t-\sigma) x_{0}^{P}{ }{ }_{f}(s) d s$

(1.42.b) $x_{t}^{P_{1}}=T(t-\sigma) x_{t}^{P_{1}}+\int_{\sigma}^{t} T(t-s) x_{0}^{P_{1}} f(s) d s$

$(1.42 . c) x_{t}^{Q}=T(t-\sigma) x_{t}^{Q}+\int_{\sigma}^{t} T(t-s) x_{0}^{Q} f(s) d s$

Seja $B\left(R^{+}\right)=\left\{f: R^{+} \rightarrow R^{n}:\right.$ f é contínua e limitada em $\left.\mathrm{R}^{+}\right\}$com a norma

$$
\left.|| f\right|_{B\left(R^{+}\right)}=\sup _{t \in R^{+}}|f(t)| \text {. }
$$

Definindo $B\left(R^{-}\right), \quad B(R)$ de maneira similar e $P_{\omega}=\left\{f: R \rightarrow R^{n}\right.$ : $f \quad \bar{e}$ continua e w-periödica\} coma norma

$$
|| f||_{\omega}=\sup _{0 \leq t \leq \omega}|f(t)|
$$

temos que estes espaços são espaços de Banach com as respectivas normas.

Daremos a seguir uma condição necessāria para que a equação (1.40) tenha uma solução em $B$ para $f \in B$, onde $B$ é um dos espaços acima.

\section{Lema 1.31}

Se $B$ é um dos espaços acima e a equação (1.40) tem uma solução em $B$ para cada $f \in B$ então não existem autovalo res da equação característica associada a equação (1.39) sobre - eixo imaginärio, isto $\vec{e}, \Lambda_{0}=\emptyset$ e portanto $\mathrm{P}_{0}=\{0\}$.

\section{Demonstração}

Suponhamos que a equação $(1.40)$ tem uma solução em $B$ para cada $f \in B$ e que $\Lambda_{0} \neq \emptyset$, isto $\dot{e}$, existe $\lambda_{0} \in \Lambda_{0}$. Mostre 
mos que isto nos leva a uma contradição. Para isto, seja C de composto por $\Lambda$ como anteriormente, isto $\bar{e}, C=P_{0} \oplus P_{1} \oplus Q e$ $\Phi_{0}$ base para $P_{0}, \Psi_{0}$ base para o auto-espaço generalizado da equação adjunta associada com. $\Lambda_{0}$, tal que. $\left(\Psi_{0}, \Phi_{0}\right)=I$ e seja $B$ uma matriz tal que $A \Phi_{0}=\Phi_{0} B$, onde $A$ e o geradorin finitesimal associado com a equação (1.39). Observemos que os autovalores de $B$ coincidem com os elementos de $\Lambda_{0}$, logo têm parte real igual a zero. Mostraremos que existe $f \in B$ tal que toda solução da equação (1.42.a) é não limitada, contradizendo a hipötese do Lema.

Se $x_{t}^{P_{0}}=\Phi_{0} z(t)$, temos que $z(t)$ satisfaz

(1.43) $\dot{z}(t)=B z(t)+\Psi_{0}(0) E(t)$.

Seja $a \in R^{n} \backslash\{0\}$ tal que $a B=\lambda_{0} a$. Temos que $a \Psi_{0}(0) \neq 0$, pois, do contrārio, $a e^{-B(t+s)} \Psi_{0}(0)=a e^{-B t_{\Psi}(s)}$, $0 \leq s \leq r$ seria uma solução, não nula, da equação adjunta com condição inicial nula. Logo,

$$
a \dot{z}(t)=\lambda_{0} a z(t)+a \Psi_{0}(0) f(t)
$$

Tomando-se, $f(t)=\left[a \Psi_{0}(0)\right]^{\prime}$, onde, representa a transposta, temos que $f \in B$ e teremos

$(1.44) \quad a \dot{z}(t)=\lambda_{0} a z(t)+\left(a \Psi_{0}(0)\right)\left(a \Psi_{0}(0)\right)^{\prime}$

Se $\omega(t)=\operatorname{az}(t)$ então $\omega(t)$ satisfaz $(1.44)$, isto $\bar{e}, \quad \dot{\omega}(t)=\lambda_{0} \omega(t)+c$, onde $c=\left(a \Psi_{0}(0)\right)\left(a \Psi_{0}(0)\right) \cdot \neq 0$ e por tanto $\omega(t)=\alpha e^{\lambda_{0} t}+c t$, onde $\lambda_{0} \vec{e}$ imaginärio poro. Logo, $|a z(t)|=|\omega(t)| \rightarrow \infty$ quando $t \rightarrow \infty$ ou $t+-\infty$, isto é, $|a z(t)|$ é não limitada, conseqüentemente cada solução da equação (1.43) é não limitada para a particular f tomada. Logo concluímos que 
$x_{t}^{P_{0}} \bar{e}$ não limitada, o que é um absurdo, portanto $\Lambda_{0}=\emptyset$ e $P_{0}=\{0\}$.

\section{$\underline{\text { Lema } 1.32}$}

Suponhamos que $\Lambda_{0}=\emptyset, B$ é um dos espaços definidos anteriormente e $\pi: B \rightarrow B$ uma projeção continua de $B$ sobre as soluções da equação (1.39) que estão em B. Então

$$
\begin{aligned}
& (1.45 . a) \pi B\left(R^{-}\right)=\left\{g: R^{-} \rightarrow R^{n} \text { : existe } \phi \in P_{I} \operatorname{com} g(t)=T(t) \phi(0), t \leq 0\right\} \\
& (1.45 . b) \pi B\left(R^{+}\right)=\left\{g: R^{+} \rightarrow R^{n} \text { : existe } \phi \in Q \text { com } g(t)=T(t) \phi(0), t>0\right\} \\
& \text { (1.45.c) } \pi B(R)=\{0\}
\end{aligned}
$$

Alēm disso, para toda $f \in B$, existe uma ünica solução Kf da equação (1.40) tal que $\pi K f=0$ e $K: B \rightarrow B$ é um operador linear continuo.

\section{Demonstração}

Consideremos o caso em que $B=B\left(R^{-}\right)$. Logo $\pi B\left(R^{-}\right)$ contēm o conjunto dado pela expressão (1.45.a), pois $g(t)=$ $=T(t) \phi(0)=x(\phi)(t)$, portanto segue do Teorema 1.30 que é uma solução limitada em $R^{-}$. Para mostrar que o conjunto dado pela expressão (1.45.a) contēm $\pi B\left(R^{-}\right)$basta supor, por absurdo, que existe $\phi \in Q, \phi \neq 0$, tal que $x_{t}(\phi)$ è uma solução da equação (1.39), em $Q$, limitada em $R^{-}$e verificar que $x_{t}(\phi)=0$ para $t \leq 0$. De maneira anāloga mostra-se (1.45.b) e (1.45.c). Suponhamos que $f \in B$ e $x=x(\phi, f)$ uma solução da $\underline{e}$ quação (1.40) em $B$, com condição inicial $\phi$ emzero, isto é, $x_{0}(\phi, f)=\phi$. Além disso, seja $\pi_{P_{I}}: C \rightarrow P_{1}$ uma projeção de $C$ sobre $P_{1}$, definida pela decomposição $C=P_{1} \oplus Q$, isto é, 
se $\phi \in C$ então $\phi=\phi^{P} 1+\phi^{Q}$, logo $\pi_{P_{1}} \phi=\phi^{P}$. Temos que (1.46) $x_{t}^{P_{1}}=T(t) \phi^{P_{1}}+\int_{0}^{t} T(t-s) x_{0}^{P^{1}} f(s) d s$

(1.47) $x_{t}^{Q}=T(t-\sigma) x_{\sigma}^{Q}+\int_{\sigma}^{t} T(t-s) x_{0}^{Q} f(s) d s \quad$ para $\sigma \leq t \leq 0$

Sejam $k, \alpha$, nümeros reais positivos dados pelo Teorema 1.30 , tal que

(1.48.a) $\left|\mathrm{T}(t) \phi^{\mathrm{P}}{ }^{1}\right| \leq \mathrm{Ke}^{\alpha t}\left|\phi^{\mathrm{P}}\right|,\left|\mathrm{T}(t) \mathrm{X}_{0}^{\mathrm{P}}\right| \leq \mathrm{Ke}^{\alpha t}, \quad t \leq 0$

(1.48.b) $\left|T(t) \phi^{Q}\right| \leq K e^{-\alpha t}\left|\phi^{Q}\right|,\left|T(t) x_{0}^{Q}\right| \leq K e^{-\alpha t}, \quad t \geq 0$

Logo pelas expressões $(1.47)$ e (1.48.b) obtemos, $\left|x_{t}^{Q}-\int_{\sigma}^{t} T(t-s) x_{\sigma}^{Q} f(s) d s\right| \leq K e^{-\alpha(t-\sigma)}\left|x_{\sigma}^{Q}\right|$ para $\sigma \leq t \leq 0$

Como $\left|x_{\sigma}^{Q}\right|$ é limitada para $\sigma \leq 0$, podemos comar o limite quando $\sigma \rightarrow-\infty$ na expressão acima e obter

$\lim _{\sigma \rightarrow-\infty}\left|x_{t}^{Q}-\int_{\sigma}^{t} T(t-s) x_{o}^{Q} f(s) d s\right|=0, \quad t \leq 0$

Portanto,

(1.49)

$$
x_{t}^{Q}=\int_{-\infty}^{t} T(t-s) x_{0}^{Q} f(s) d s
$$

$$
\left|x_{t}^{Q}\right| \leq \frac{K}{\alpha} \sup _{u \leq 0}|f(u)|
$$

\section{Logo}

(1.50) $\quad x_{t}(\phi, f)=T(t) \phi^{P}+\int_{0}^{t} T(t-s) x_{0}{ }^{1} f(s) d s+$

$$
+\int_{-\infty}^{t} T(t-s) X_{0}^{Q_{f}} f(s) d s, \quad t \leq 0
$$


Us ando $(1.48 . a)$, obtemos

(1.51) $\left|\mathrm{T}(t) \phi^{\mathrm{P}}{ }^{1}+\int_{0}^{t} \mathrm{~T}(t-s) \mathrm{X}_{0}^{\mathrm{P}^{1}} f(s) \mathrm{d} s\right| \leq$

$$
\leq \mathrm{Ke}^{\alpha t}\left|\phi^{\mathrm{P}} 1\right|+\frac{\mathrm{K}}{\alpha} \underset{\mathrm{u}<0}{\sup }|\mathrm{f}(\mathrm{u})|
$$

para $t \leq 0$ e $\phi^{P} \in P_{1}, \log 0 x(\phi, f) \in B$

Reciprocamente, a expressão (1.50) é uma solução da equação (1.40) em $B$ satisfazendo as estimativas (1.49) e (1.51). Seja $\quad x=x\left(\phi^{P}, f\right)$ definida pela expressão (1.50) e seja $k f=(I-\pi) x$ onde $\pi: B \rightarrow B$ é uma projeção de $B$ sobre as soluções da equação (1.39) que estão em $B$. Então $K: B \rightarrow B$ e pode ser dada por $K f=(I-\pi) \times(0, f)$. Portanto, linear conti nua e satisfaz a equação (1.40).

o operador $K$ pode ser dado explicitamente por

$$
(K f)_{t}=\int_{0}^{t} T(t-s) x_{0}^{P} f(s) d s+\int_{-\infty}^{t} T(t-s) x_{0}^{Q} f(s) d s, \quad t \leq 0 \text {. }
$$

De maneira semelhante mostra-se que o resultado é verdadeiro para o caso em que $f \in B\left(R^{+}\right)$ou $B(R)$.

Para o casode $f \in B\left(R^{+}\right)$, obtemos a seguinte expressão para K:

$$
(K f)_{t}=\int_{\infty}^{t} T(t-s) X_{0}^{P}{ }^{1} f(s) d s+\int_{0}^{t} T(t-s) x_{0}^{Q_{f}(s) d s, \quad t \geq 0}
$$

e para o caso de $f \in B(R)$, obtemos:

$$
(K f)_{t}=\int_{\infty}^{t} T(t-s) x_{0}^{P}{ }^{1} f(s) d s+\int_{-\infty}^{t} T(t-s) x_{0}^{Q_{f}} f(s) d s, t \in R .
$$


o próximo resultado nos darā uma condição necessária e suficiente para que a equação (1.40) tenha uma solução w-periódica sendo o termo forçante, f, $\omega$-periódico.

Para isso, consideremos $\tilde{\pi}: P_{\omega} \rightarrow P_{\omega}$ uma projeção con tinua de $P_{\omega}$ sobre as soluções $\omega$-periödicas da equação (1.39). Por exemplo, podemos definir $\tilde{\pi}$ da seguinte maneira. Seja $U=\left(\phi_{1}, \ldots, \phi_{d}\right)$ base para as soluções w-periódicas da equação (1.39), seja $U^{\prime}$ a transposta de $U$ e definamos

(1.52) $\quad \tilde{\pi} f=U\left[\int_{0}^{\omega} U^{\prime}(s) U(s) d s\right]^{-1} \int_{0}^{\omega} U^{\prime}(s) f(s) d s$

é fäcil verificar que $\tilde{\pi}$ satisfaz as condições acima.

Teorema 1.33 (Alternativa de Fredholm)

Se $f \in P_{\omega}$ então a equação (1.40) tem uma solução em $P_{\omega}$ se e somente se

(1.53) $\quad \int_{0}^{\omega} y(t) f(t) d t=0$

para toda y solução w-periódica da equação adjunta

$(1.54) \quad \dot{y}(s)=-\int_{-r}^{0} \operatorname{dn}(\theta) y(s-\theta)$.

Além disso, existe uma projeção contínua, $J: P_{\omega} \rightarrow P_{\omega}$ tal que o conjunto das $f \in P_{\omega}$ que satisfazem a relação (1.53) $\bar{e}$ exatamente $(I-J) P_{\omega}$ e existe uma transformação linear continua $K:(I-J) P_{\omega} \rightarrow(I-\tilde{\pi}) P_{\omega}$ tal que $K f$ é uma solução da equa ção (1.40) para cada $f \in(I-J) P_{\omega}$.

\section{Demonstração}

Consideremos a decomposição de $C$ como $C=P_{0} \oplus P_{1} \oplus Q$, 
como no início desta seç̧ão. Em virtude do Lema 1.32 , as equações $(1.42 . b)$ e (1.42.c) tem uma ünica solução w-periódica para $f \in P_{\omega}$ e são dadas explicitamente por

$$
x_{t}^{P}{ }^{P}=\int_{\infty}^{t} T(t-s) x_{0}^{P^{1}} f(s) d s
$$

$$
x_{t}^{Q}=\int_{-\infty}^{t} T(t-s) x_{0}^{Q} f(s) d s
$$

Logo, basta considerarmos somente a equação (1.42.a). Mantendo as notações da prova do Lema 1.31 , se $\Phi_{0}$ é uma base para $P_{0} e$ $B$ é definida pela relação $A \Phi \Phi_{0}=\Phi_{0} B$, onde $A \bar{e}$ - gerador infinitesimal associado a equação (1.39), então se $x_{t}^{P^{0}}=\Phi_{0} z(t)$, teremos que $z(t)$ será solução da equação (1.43). E um fato conhecido da Teoria de Equações Diferenciais ordinárias que a equação (1.43) tem uma solução w-periōdica se e somente se

$$
\int_{0}^{\omega} u(t) \Psi_{0}(0) f(t) d t=0
$$

para toda solução, u, w-periódica da equação adjunta $\dot{u}(t)=$ $=-u(t) B$. Como referência para este resultado veja $[5$; pag. 146].

$$
\begin{aligned}
& \text { Isto é, } \\
& \int_{0}^{\omega} u_{0} e^{-B t_{\Psi}}(0) f(t) d t=0
\end{aligned}
$$

para toda $u_{0}$, para oqual $u_{0} e^{-B t}$ é $\omega$-periôdica. Mas $u_{0} e^{-B t_{\Psi_{0}}(0)}$, para estes $u_{0}^{\prime} s$, coincide com as soluções $\omega$-perió dicas da equação adjunta (1.54), pois $u_{0} e^{-B t_{\Psi}}(0)=u_{0} \Psi_{0}(t)$, e isto completa a primeira parte da prova do Teorema 1.33 .

$$
\text { Para a segunda parte, seja } v=\operatorname{col}\left(\psi_{1}, \ldots, \psi_{d}\right) \text { base }
$$
para as soluções w-periódicas da equação (1.54) e definamos 
$J: P_{\omega} \rightarrow P_{\omega}$ como

(1.56) Jf $=V^{\prime}\left[\int_{0}^{\omega} V(s) V^{\prime}(s) d s\right]^{-1} \int_{0}^{\omega} V(s) f(s) d s$

onde $V^{\prime}$ é a transposta de $V$.

E simples mostrar que $J$ satisfaz as condições requeridas pelo Teorema 1.33. Definindo $K:(I-J) P_{\omega} \rightarrow(I-\tilde{\pi}) P_{\omega}$ por $\mathrm{Kf}=(\mathrm{I}-\tilde{\pi}) \times(f)$, com base na prova do Lema 1.32 , concluímos a prova do Teorema 1.33 . 


\section{CAPITULO 2}

Neste capitulo nós daremos um procedimento para determinar a existência de soluções periódicas de algumas classes de equações diferenciais funcionais autônomas. Para isto, falaremos na Bifurcação de Hopf e aplicaremos os teoremas de ponto fi xo ejetivo, dados no Capítulo 0. Finalmente, aplicando um teorema sobre existência de autovetores de aplicações não lineares, em um cone obteremos um resultado sobre existência de solu ções periódicas para equaçōes diferenciais com retardamento, que deixam uma esfera invariante em $R^{2}$. Este resultado tem inspi ração em [2; pag. 27], entretanto, é distinto deste ültimo.

\subsection{A Bifurcação de Hopó}

Consideremos uma família de EDFR dependendo de um parạ metro real, da forma

$$
\text { (2.1) } \quad \dot{x}(t)=F\left(\alpha, x_{t}\right)
$$

onde $F(\alpha, \phi)$ tem primeira e segunda derivadas continuas em $\alpha, \phi$, para $\alpha \in R, \phi \in C$ e $F(\alpha, 0)=0$ para todo $\alpha \in R$. Definamos $L: R \times C \rightarrow R^{n}$ por

$(2.2) \quad \mathrm{L}(\alpha) \psi=\mathrm{F}_{\phi}(\alpha, 0)$

onde $F_{\phi}(\alpha, 0)$ é a derivada de $F(\alpha, \phi)$ comrelação a $\phi$, cal culada em $\phi=0$, e consideremos $f: R \times C \rightarrow R^{n}$ dada por (2.3) $\quad f(\alpha, \phi)=F(\alpha, \phi)-L(\alpha) \phi$ para $(\alpha, \phi) \in R \times C$. 
Suponhamos que:

(H1) A $\operatorname{EDFR}(L(0))$ tem uma raiz característica simples, ima ginária pura, $\lambda_{0}=i \nu_{0} \neq 0$ e todas as raízes caracteristicas $\lambda_{j} \neq \lambda_{0}, \bar{\lambda}_{0}$, satisfazem $\lambda_{j} \neq m \lambda_{0}, m \in Z$.

Como $L(\alpha)$ é continuamente diferenciävel em $\alpha$, o. Le ma 1.22 , implica que existe $\alpha_{0}>0$ e uma raiz característica simples, $\lambda(\alpha)$, da EDFR(L $(\alpha))$ que tem derivada continua em $\alpha$ para $|\alpha|<\alpha_{0}$ e $\lambda(0)=\lambda_{0}$.

Suponhamos que:

(H2) $\operatorname{Re} \lambda^{\prime}(0) \neq 0$.

Tomando-se $\alpha_{0}$, suficientemente pequeno, podemos supor que $\operatorname{Im} \lambda(\alpha) \neq 0$ para $|\alpha|<\alpha_{0}$. Seja $\phi_{\alpha} \in$ C base para - auto-espaço generalizado, $P(\alpha)$, associado a $\lambda(\alpha)$, da $\operatorname{EDFR}(L(\alpha))$. Observemos que

$$
\left(\operatorname{Re} \phi_{\alpha}, \operatorname{Im} \phi_{\alpha}\right) \stackrel{\operatorname{def}}{=} \Phi_{\alpha}
$$

forma uma base para o auto-espaço generalizado da EDFR(L $(\alpha))$ as sociado a $\lambda(\alpha), \quad \bar{\lambda}(\alpha)$. Da mesma maneira, obtemos $\Psi_{\alpha}$, base para o auto-espaço da equação ajunta formal, com $\left(\Psi_{\alpha}, \Phi_{\alpha}\right)=I=\underline{i}$ dentidade.

De acordo com o Téorema 1.20 e o Lema 1.29 , existe uma matriz $B(\alpha), 2 \times 2$, continuamente diferenciävel, tal que (2.4) $\Phi_{\alpha}(\theta)=\Phi_{\alpha}(0) e^{B(\alpha) \theta}, \quad-r \leq \theta \leq 0$, onde os ünicos autovalores de $B(\alpha)$ são $\lambda(\alpha)$ e $\bar{\lambda}(\alpha)$. Por uma mudança de coordenadas e redefinindo o parảmetro $\alpha$, se necessário, podemos assumir que 


$$
B(\alpha)=v_{0} B_{0}+\alpha B_{1}(\alpha)
$$

onde $B_{0} E\left[\begin{array}{cc}0 & 1 \\ -1 & 0\end{array}\right]:$ e $B_{1}(\alpha)=\left[\begin{array}{cc}1 & \gamma(\alpha) \\ -\gamma(\alpha) & 1\end{array}\right], \quad \cos \gamma(\alpha)$ continuamente diferenciável para $|\alpha|<\alpha_{0}$. De fato, como a $\underline{i}$ gualdade (2.5) deve ser verificada, a menos de mudança de variä veis, basta demonstrarmos que existe $\gamma(\alpha)$, de modo que as matrizes do primeiro e do segundo membro de (2.5) tenham mesmo polinômio característico. Ou seja,

$$
\lambda(\alpha) \bar{\lambda}(\alpha)=\alpha^{2}+\left[\nu_{0}+\alpha \gamma(\alpha)\right]^{2}
$$

que define uma possivel escolha para $\gamma(\alpha)$.

Mostraremos a seguir que as hipóteses (H1) e (H2) imo plicam que a equação (2.1) tenha soluções periódicas, não nulas, para a pequeno, com período próximo a $2 \pi / \nu_{0}$.

\section{Teorema 2.1 (Bifurcação de Hopf)}

Suponhamos que $F(\alpha, \phi)$ tenha primeira e segunda deri vadas continuas com respeito a $\alpha, \phi, F(\alpha, 0)=0$, para todo $\alpha \in R$, e que (H1) e (H2) estão satisfeitas. Então existem cons tantes $a_{0}, \alpha_{0}, \delta_{0}>0$; funçōes $\alpha(a) \in R, \omega(a) \in R \quad$ uma função $x *(a), \omega(a)$-periódica, todas continuamente diferenciáveis para $|a|<a_{0}$, tal que $x^{*}(a)$ é solução da equação (2.1) satisfazendo

(2.6) $\quad x_{0}^{*(a)}{ }^{P} \alpha(a)=\Phi_{\alpha(a)} y_{0}^{*(a)}$ e $x_{0}^{*}(a){ }^{Q} \alpha(a)=z_{0}^{*}(a)$ onde $y_{0}^{*}(a)=\operatorname{col}(a, 0)+o(|a|), \quad z_{0}^{*}(a)=o(|a|)$, quando $|a| \rightarrow 0$. Além disso, para $|\alpha|<\alpha_{0},\left|\omega-2 \pi / \nu_{0}\right|<\delta_{0}$, cada solução $x$, $w$-periödica, da equação $(2.1), \operatorname{com}\left|x_{t}\right|<\delta_{0}$, deve ser do tipo 
acima, exceto por defasagem.

\section{Demonstração}

Seja $B \in\left(-1,1, \omega_{0}=2 \pi / \nu_{0}\right.$ e definamos

$u(\tau)=x((1+\beta) \tau), \tau \geq 0$, onde $x$ é uma solução da equação (2.1), e seja $u_{\tau, \beta}(\theta)=u(\tau+\theta /(1+\beta))$ para $-r \leq \theta \leq 0$. Com is to, temos que a equação $(2.1)$ é equivalente a equação

(2.7) $\frac{d u(\tau)}{d \tau}=(1+B) F\left(\alpha, u_{\tau, \beta}\right)$.

Se esta equação tiver uma solução $\omega_{0}$-perijóca, então a equação (2.1) terá uma solução $(1+\beta) \omega_{0}$-periódica e reciproca mente.

Reescrevendo a equação (2.7), obtemos:

(2.8) $\frac{\mathrm{du}(\tau)}{\mathrm{d} \tau}=\mathrm{L}(0) \mathrm{u}_{\tau}+\mathrm{N}\left(B, \alpha, u_{\tau}, u_{\tau, \dot{j}}\right)$

onde $N\left(\beta, \alpha, u_{\tau}, u_{\tau, \beta}\right)=(1+\beta) L(\alpha) u_{\tau, \beta}-L(0) u_{\tau}+(1+\delta) f\left(\alpha, \alpha_{\tau, \beta}\right)$.

Isto significa que podemos considerar a equação (2.8)

como uma perturbação da equação linear autônoma

(2.9) $\quad \frac{d u(\tau)}{d \tau}=L(0) u_{\tau}$.

Sabemos que as colunas $U(\tau)=\omega_{0}(0) e^{B(0) \tau}$, ut $\in R$, formam uma base para as soluções u periódicas da eyuação (2.9) e as linhas de $V(\tau)=e^{-B(0) \tau_{\psi}}{ }_{0}(0), \quad \tau \in R$, formam uma base

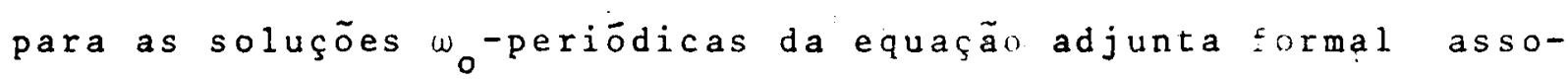
ciada à equação (2.9).

Podemos aplicar o Teorema 1.33 , para obcer condições necessārias e suficientes para a existência de soluções $\omega_{0}$-pe riödicas da equação (2.8). Antes porēm, é conveniente observar que se $u(\tau)$ é solução da equação (2.9) então $u^{h}(\tau)$ dẹf $_{u(\tau+h)}$ 
também serā uma solução da equação (2.9). A solução geral de $(2.9) \bar{e}$

$$
u(\tau)=U(\tau)\left(\begin{array}{l}
x \\
y
\end{array}\right) \quad \text { onde } x, y \in R \text {. }
$$

Afirmamos que existem a, $h \in R$ tal que

$$
U(\tau+h)\left(\begin{array}{l}
x \\
y
\end{array}\right)=U(\tau) \cos (a, 0), \quad \forall \tau \in R .
$$

De fato,

$$
U(\tau+h)\left(\begin{array}{l}
x \\
y
\end{array}\right)=U(\tau) e^{B(0) h}\left(\begin{array}{l}
x \\
y
\end{array}\right)
$$

logo, basta mostrar que existem $a, h \in R$ tal que

$$
e^{B(0) h}\left(\begin{array}{l}
x \\
y
\end{array}\right)=\operatorname{col}(a, 0)
$$

Temos que

$$
e^{B(0) h}\left(\begin{array}{ll}
x \\
y
\end{array}\right)=\left[\begin{array}{cc}
e^{i \nu_{0} h} & 0 \\
0 & e^{-i \nu_{0} h}
\end{array}\right]\left(\begin{array}{l}
x \\
y
\end{array}\right)
$$

e como queremos soluções reais teremos

$$
\begin{aligned}
e^{B(0) h}\left(\begin{array}{l}
x \\
y
\end{array}\right) & =\left[\begin{array}{cc}
\cos \nu_{0} h & \operatorname{sen} \nu_{0} h \\
-\operatorname{sen} \nu_{0} h & \cos \nu_{0} h
\end{array}\right]\left(\begin{array}{l}
x \\
y
\end{array}\right)= \\
& =\left(x \cos \nu_{0} h+y \operatorname{sen} \nu_{0} h,-x \operatorname{sen} \nu_{0} h+y \cos \nu_{0} h\right) .
\end{aligned}
$$

Assim o problema se resume em mostrar a existência de soluções $h$. de $-x \operatorname{sen} \nu_{0} h+y \cos \nu_{0} h=0$, que é um fato óbvio. Portanto, uma aplicação direta do Teorema 1.33, nos mostra que cada solução $\omega_{0}$-periódica da equação (2.8), exceto por uma defasagem, é 
solução. das equações:

$(2.10 . a) u(\tau)=U(\tau) \operatorname{col}(a, 0)+K(I-J) N(\beta, \alpha, u,, u,, \beta)(\tau)$

$(2.10 . b) \operatorname{JN}(\beta, \alpha, u,, u, \beta)=0$

e reciprocamente, onde $K$, J são dadas no Teorema 1.33 .

Aplicando-se o teorema da função implícita a equação (2.10.a) obtemos $u *=u *(a, \beta, \alpha)$, para a, $\beta, \alpha$ numa vizinhan ça suficientemente pequena de zero, continuamente diferenciável em a, $B, \alpha$ e satisfazendo

(2.11) $u *(a, 0,0)-U(\cdot) \cos (a, 0)=o(|a|)$ quando $|a|+0$.

Como $u *(\tau)=u *(a, \beta, \alpha)(\tau)$ satisfaz a equação $(2.10 . a)$ ela também satisfaz a equação integral associada a (2.7) e portanto é continuamente diferenciável em $\tau$.

Logo, toda solução $\omega_{0}$-periódica da equação (2.8), pode ser obtida encontrando-se $a, \beta, \alpha$, solução da equação de bifurcação

$(2.12) \quad \operatorname{JN}(\beta, \alpha, u *(a, \beta, \alpha), u *, \beta(a, \beta, \alpha))=0$.

Do Teorema 1.33, J pode ser tomada c.omo $J(h)=V\left[\int_{0}^{\omega_{0}} V(s) V^{\prime}(s) d s\right]^{-1} \int_{0}^{\omega_{0}} V(s) h(s) d s$, onde $h \in P_{\omega_{0}}$.

Logo, (2.12) é equivalente a equação:

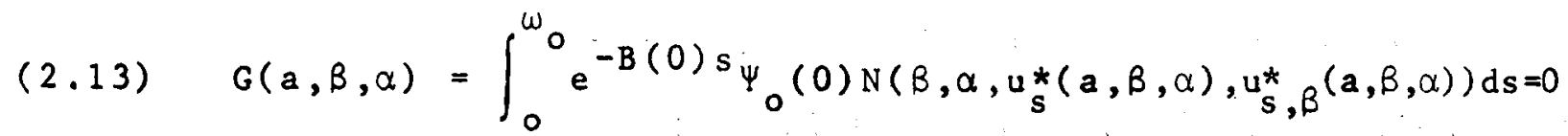

Portanto, basta resolver a equação $G(a, \beta, \alpha)=0$.

Observemos que $G(0, \beta, \alpha)=0$ para todo $\beta, \alpha$. 
Seja $H(a, \beta, \alpha)=G(a, \beta, \alpha) / a$ se $a \neq 0$ e $H(0, \beta, \alpha)=$ $=\lim _{a \rightarrow 0} \frac{G(a, b, a)}{a}$. Com isto temos que

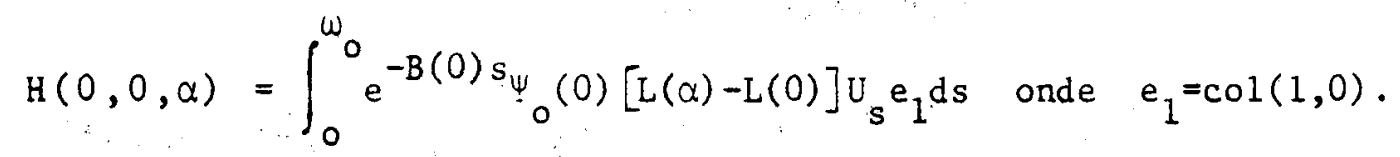

Calculando a derivada com relação a a no ponto $(0,0,0)$ e usando o Lema 1.29 , obtemos:

$$
\frac{\partial H(0,0,0)}{\partial \alpha}=\omega_{0}\left[\begin{array}{c}
+1 \\
-\gamma(0)
\end{array}\right] .
$$

Além disso, um simples cálculo, nos mostra que:

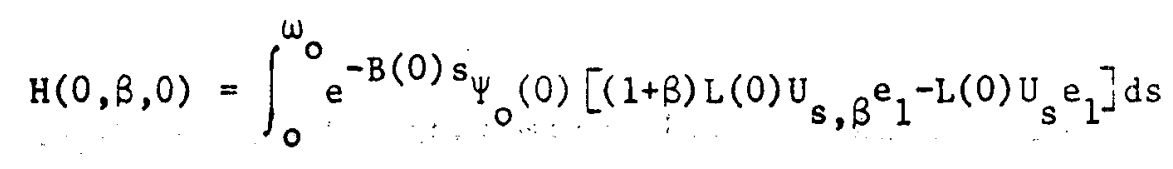

Escrevendo-se esta integral como a diferença de duas integrais, fazendo uma mudança da variāvel $s$ para $s /(1+B)$ na primeira integral e notando que

$$
\frac{\partial U(s /(1+\beta))}{\partial(s /(1+\beta))}=L(0) U_{s /(1+B), \beta}
$$

teremos,

$$
H(0, B, 0)=B \int_{0}^{\omega} e^{-B(0) s_{\Psi_{0}}(0) \Phi} \Phi_{0}(0) e^{B(0) s_{B}(0) e_{1} d s .}
$$

Se $x$ é uma solução de (2.9) e y é uma solução da e quação adjunta de $(2.9)$ então vimos que $\left(y^{t}, x_{t}\right)=$ cte para to do $t$, portanto 


$$
\begin{aligned}
& I=\left(e^{-B(0)(s+\cdot)} \Psi_{0}(0), \Phi_{0}(0) e^{B(0)(s+\cdot)}\right)=
\end{aligned}
$$

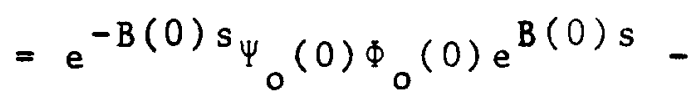

$$
\begin{aligned}
& -\int_{-r}^{0} d n(\theta) \int_{0}^{\theta} e^{-B(0)(s+\xi-\theta)} \Psi_{0}(0) \Phi_{0}(0) e^{B(0)(s+\xi)} d \xi
\end{aligned}
$$

para todo $s \in R$.

Integrando ambos os membros de 0 a $\omega_{0}$ e usando o fato que a segunda integral do segundo membro da igualdade é zero, obtemos:

$$
\omega_{0} I=\int_{0}^{\omega_{0}} e^{-B(0) s_{\psi}}(0) \Phi_{0}(0) e^{B(0) s} d s .
$$

Multiplicando-se ambos os membros desta igualdade $B B(0) e_{1}$ a direita, obtém-se

$$
\begin{aligned}
& B \omega_{0} B(0) e_{1}= \\
& =B \int_{0}^{\omega} e^{-B(0) s_{\Psi}}(0) \Phi_{0}(0) e^{B(0) s_{B}} B(0) e_{2} d s=H(0, B, 0) \\
& \text { Portanto, } \\
& H(0, B, 0)=B \omega_{0} B(0) e_{1} . \\
& \text { Logo temos: } \\
& H(0,0,0)=0 \\
& \text { e } \quad \frac{\partial H}{\partial(B, \alpha)}(0,0,0)=\left(\omega_{0} B(0) e_{1}+\omega_{0} B_{1}(0) e_{1}\right)= \\
& =\omega_{0}\left[\begin{array}{cc}
0 & +1 \\
-\nu_{0} & -\gamma(0)
\end{array}\right] \text {, }
\end{aligned}
$$


donde segue que $\operatorname{det}\left(\frac{\partial H}{\partial(\beta, \alpha)}(0,0,0)\right)=+v_{0} \neq 0$.

Portanto pelo teorema da função implícita segue que e xistem funções $B(a)$ e $\alpha(a)$, continuamente diferenciäveis com $B(0)=\alpha(0)=0, \operatorname{com} H(a, \alpha(a), \beta(a))=0$, e esta solução é única para a numa vizinhança de zero, $|a|<a_{0}$. Logo te mos que $G(a, \beta(a), \alpha(a))=0$ e portanto $u *(a)=u *(a, \beta(a), \alpha(a))$ é solução $w_{0}$-periódica da equação (2.8).

Observemos que $x^{*}(a)$, correspondente a $u^{*}(a)$, serä uma solução da equação (2.1), $w(a)$-periódica, onde $\omega(a)=(1+B(a)) \omega_{0}$.

$$
\begin{aligned}
& \text { Se } x_{0}^{*(a)}{ }^{P} \alpha(a)=\Phi_{\alpha(a)} y *(a), \quad \text { como } \\
& x_{0}^{*}(a)(\theta)=u_{0, \beta(a)}^{*}(a, \beta(a), \alpha(a))(\theta),-r \leq \theta \leq 0, \text { temos que } \\
& \lim _{|a| \rightarrow 0} \frac{x_{a}^{*}(a)}{|a|}=\lim _{|a| \rightarrow 0} \Phi(a) \lim _{|a| \rightarrow 0} \frac{y^{*}(a)}{|a|}= \\
& =\Phi(0) \lim _{|a| \rightarrow 0} \frac{y *(a)}{|a|}=U \lim _{|a| \rightarrow 0} \frac{y *(a)}{|a|}
\end{aligned}
$$

e

$$
\lim _{|a| \rightarrow 0} \frac{x_{0}^{*}(a)}{|a|}=\lim _{|a| \rightarrow 0} \frac{u_{o, \beta(a)}^{*}(a, \beta(a), \alpha(a))^{P_{\alpha(a)}}}{|a|}=U e_{1}
$$

então temos que $y *(a)=\operatorname{col}(a, 0)+o(|a|)$ quando $|a|+0$.

Além disso, se $x_{0}^{*}(a)^{Q} \alpha(a)=z_{0}^{*}(a)$ então

$$
\lim _{|a| \rightarrow 0} \frac{x_{0}^{*}(a) Q_{\alpha(a)}}{|a|}=\lim _{|a| \rightarrow 0} \frac{u_{0, B(a)}(a, B(a), \alpha(a))^{Q_{\alpha(a)}}}{|a|}=0
$$

Logo $z_{0}^{*}(a)=O(|a|)$ quando $|a| \rightarrow 0$.

Por ültimo, se $x$ é uma solução w-periódica da equa$\oint^{a} \tilde{o}_{0}(2.1) \operatorname{com}\left|\omega-\omega_{0}\right|<\delta_{0},|\alpha|<\alpha_{0}$ e $\left|x_{t}\right|<\delta_{0}$ temos da 
unicidade das funções $\alpha(a), \quad \beta(a)$ para $|a|<a_{0}$ e dos au tovalores da EDFR(L(0)) não serem múltiplos inteiros de $\lambda_{0} i \underline{\text { I }}$ plicam que a solução $x$ será do tipo mencionado acima, exceto por uma defasagem.

2.2. Teoremas sobre Periodicidade de EOFR Autōnomas Envolvendo Ponto Ejetivo

A seguir, daremos um teorema sobre periodicidade, utilizando os teoremas 0.4 e 0.5 , que envolvem o conceito de ponto ejetivo, para uma EDFR autônoma.

Consideremos as equações

$(2.14) \quad \dot{x}(t)=L\left(x_{t}\right)+f\left(x_{t}\right)$

$(2.15) \quad \dot{y}(t)=L\left(y_{t}\right)$

onde $L: C \rightarrow R^{n}$ é linear e continua; $f: C \rightarrow R^{n} \bar{e}$ completa mente continua, $f^{\prime}$ é continua e $f(0)=f^{\prime}(0)=0$.

Para cada autovalor, $\lambda$, associado a equação (2.15) e xiste uma decomposição de $C$ como $C=P_{\lambda} \oplus Q_{\lambda}$, onde $P_{\lambda} e$ $Q_{\lambda}$ são invariantes pela aplicação da solução associada a equa ção (2.15), $T(t) \phi=y_{t}(\phi)$ para $\phi \in c, t \geq 0$. Sejam $T_{\lambda}$ e $\left(I-\pi_{\lambda}\right)$ as projeções definidas pela decomposição de $C$, coma imagem de $\pi_{\lambda}$ sendo $P_{\lambda}$.

Suponhamos que exista um subconjunto $K \subset C$ tal que cada solução $x(\phi)$ da equação (2.14) para $\phi \in K$, "volte" para $K$, em algum tempo $\tau(\phi)>0$, isto $\vec{e}, x_{\tau(\phi)}(\phi) \in K$, se $\phi \in K$. Logo temos definida uma aplicação $A: K+K$ dada por 
$A(\phi)=x_{\tau(\phi)}(\phi)$, para $\phi \in K$.
$\quad$ Se A é completamente continua e $K$ for fechado, Iimi tado e convexo então segue do Teorema do Ponto Fixo de Schauder, a existência de $\phi \in K$ tal que $A \phi=\phi$, isto é, uma solução periódica da equação (2.14). Para que esta solução não seja trivial necessitamos que o ponto fixo de $A$ não seja $\phi=0$. Para que isto ocorra, bastara que $\phi=0$ seja um ponto ejetivo de A em K. Logo precisaremos de um resultado que nos dê uma condi ção necessária para que $\phi=0$ seja um ponto ejetivo de A. Es ta condição é dada no seguinté resultado.

\section{Teorema 2.2}

Se as seguintes condições estão satisfeitas:

(i) Existe um autovalor, $\lambda$, associado a equação (2.15) sa tisfazendo: $\operatorname{Re} \lambda>0$.

(ii) Existe um subconjunto $\mathrm{K} \subset \mathrm{C}$, fechado, convexo tal que $0 \in K$ e existe $\delta>0$ tal que

$\nu=\nu(\delta)=\inf \left\{\left|\pi_{\lambda} \phi\right|: \phi \in \mathrm{K} \quad \mathrm{e}|\phi|=\delta\right\}>0$.

(iii) Existe uma função completamente contínua $\tau: K \backslash\{0\} \rightarrow[\alpha, \infty)$, $\alpha \geq 0$, tal que a aplicação $A: K \backslash\{0\} \rightarrow C$ dada por $A(\phi)=x_{\tau(\phi)}(\phi)$, para $\phi \in K \backslash\{0\}$, leva $K \backslash\{0\}$ em $\mathrm{K}$ e $\bar{e}$ completamente continua.

Então 0 é um ponto ejetivo de A.

\section{Demonstração}

seja $\Phi_{\lambda}=\left(\phi_{\lambda}^{1}, \phi_{\lambda}^{2}, \ldots, \phi_{\lambda}^{d}\right)$ uma base para $P_{\lambda}$ e $\pi_{\lambda} \phi=\phi_{\lambda} b$ onde $b=b(\phi) \in R^{d}$.

Para $V: C \rightarrow R$ continua, seja 
$\dot{V}(\phi)=\lim _{t \rightarrow 0^{+}} \inf \frac{1}{t}-\left[V\left(x_{t}(\phi)\right)-V(\phi)\right]$

Por um resultado de [5; pag. 232] temos que existe uma forma quadrätica definida positiva, $V(\phi)=b$ ! Bb, com a proprie dade que para todo $p>0$, existe $\delta_{0}>0$ tal que para todo $\delta$, $0<\delta<\delta_{0}, \dot{V}(\phi)>0$ se $\mathrm{V}(\phi) \geq \mathrm{p}^{2} \delta^{2}$ para $\phi \in \overline{\mathrm{B}}_{\delta}=\{\phi \in C:|\phi| \leq \delta\}$. Com a função $V(\phi)$, temos que a condição (ii) implica

que

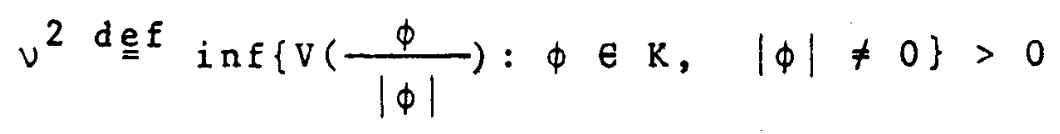

pois se $\phi \neq 0$ temos que $b=b(\phi) \neq 0, \quad$ logo

$$
\begin{aligned}
0<\left|\pi_{\lambda} \phi\right| & =|\Phi \mathrm{b}| \leq|\Phi||\mathrm{b}|=\frac{|\Phi|}{|\mathrm{b}|}|\mathrm{b}|^{2}= \\
& =\alpha|\mathrm{b}|^{2} \text { onde } \alpha=\frac{|\Phi|}{|\mathrm{b}|} .
\end{aligned}
$$

Se $B^{2}=\min \left\{c^{\prime} B c:|c|=1\right\}$, e $\phi \neq 0$ teremos

$$
V(\phi)=b^{\prime} B b=\left|b^{\prime}\right|\left[\frac{b^{\prime}}{\left|b^{\prime}\right|} B \frac{b}{|b|}\right]|b| \geq B^{2}|b|^{2} .
$$

Logo, $\quad 0<\left|\pi_{\lambda} \phi\right| \leq \alpha|b|^{2} \leq \alpha \beta^{2} v(\phi)$.

Portanto, $v^{2}>0$.

Fixemos $p<\nu$ e consideremos $\delta_{0}$, obtido acima. Lo go $V\left(x_{t}(\phi)\right)$ é crescente em $t$, enquanto $V\left(x_{t}(\phi)\right) \geq p^{2} \delta^{2}$, pa ra $\left|x_{t}(\phi)\right| \leq \delta, \quad \operatorname{com} 0<\delta<\delta_{0}$.

Seja $G(\delta)=\left\{\phi \in C:|\phi|<\delta\right.$ e $\left.V(\phi)<p^{2} \delta^{2}\right\}$. Então

$G(\delta)$ é um subconjunto aberto de $C$ e $0 \in G(\delta)$.

Como $\tau$ é completamente contínua, existe $k>0$ tal que $\tau(\phi) \leq k$, para toda $\phi \in G\left(\delta_{0}\right) \cap k$. Escolhendo $\delta_{1}$, $0<\delta_{1}<\delta_{0}$, suficientemente pequeno, de maneira que a solução $x(\phi)$ da equação (2.14) passando por $\phi \in \overline{G\left(\delta_{1}\right)}$ satisfaz 
$x_{t}(\phi) \in G\left(\delta_{0}\right)$ para: $0 \leq t \leq k$, que $\vec{e}$ uma conseqüência do teorema da continuidade das soluções em relação as condições iniciais da equação (2.14) e da continuidade de $v$.

Para toda $\phi \in G\left(\delta_{0}\right) \cap K, \operatorname{com}|\phi|=\varepsilon<\delta_{0}, \quad 0$ fato de $p<\nu$ implica que

$$
v(\phi)=V\left(|\phi| \frac{\phi}{|\phi|}\right)=|\phi|^{2}\left(\frac{\phi}{|\phi|}\right) \geq \varepsilon^{2} v^{2}>\varepsilon^{2} p^{2},
$$

logo $\dot{V}(\phi)>0$. Portanto $V(\phi)$ é crescente ao longo das soluções da equação (2.14) para $|\phi|<\delta_{0}, \phi \in K \backslash\{0\}$. Logo para $\phi \in G\left(\delta_{1}\right) \cap(k \backslash\{0\})$, existem $t_{1}>t_{2}>0$, tal que $x_{t}(\phi) \in G\left(\delta_{1}\right), \quad 0 \leq t \leq t_{2}, \quad x_{t}(\phi) \in G\left(\delta_{0}\right) \backslash G\left(\delta_{i}\right),-2^{<t<t} e_{1} e$ $x_{t_{1}}(\phi) \in \partial G\left(\delta_{0}\right)$.

Seja $n_{0}(\phi)$, o maior inteiro tal que

$A^{k} \phi \in\left\{x_{t}(\phi): 0 \leq t \leq t_{2}\right\} \subset G\left(\delta_{1}\right), \quad k=0,1, \ldots, n_{0}(\phi) . \quad$ Logo $A^{n_{0}(\phi)+1} \phi \notin G\left(\delta_{1}\right) \cap K$, mostrando que 0 é um ponto ejetivo de A .

Com isto, podemos enunciar o seguinte resultado, cuja demonstração é uma aplicação direta dos Teoremas $0.4,0.5$ e 2.2 .

\section{Teorema 2.3}

Suponhamos que $K \subset C$ é fechado convexo, $0 \in K$ e que as condições ( $i$ ), ( $i i)$, ( $i i_{i}$ ) do Teorema 2.2 estão satisfeitas. Se uma das condições abaixo ocorrer:

(iv) $K$ é limitado e de dimensão infinita

(iv') K é limitado, de dimensão finita e 0 è um ponto ex tremo de. $k$. 
(iv") Existe $M>0$, tal que $A x=\lambda x, x \in k \cap S_{M}$ implica $\lambda<I$.

Então existe uma solução periódica, não nula, da equa ção (2.14) com condição inicial em $K \backslash\{0\}$.

2.3. Soluçōes Periōdicas de EDFR Autônomas em $\mathrm{c}_{\mathrm{a}}$

Nesta secção, apresentaremos um teorema que nos garantirá a existência de soluções periódicas, não constante, em $c\left([-r, 0], S^{1}(a)\right)$, para um EDFR autônoma, quando temos uma esfé ra, $S^{1}(a)$, invariante em $R^{2}$, usando a teoria de autovetores em cones desenvolvida na secção 0.3 .

Adotaremos, a seguir, as seguintes notações:

Para a $>0$, $S^{1}(a)=\left\{x \in R^{2}:|x|=a\right\}$

e

$$
\begin{aligned}
& C_{a}=c\left([-r, 0], S^{1}(a)\right) \\
& \text { Dados } k_{1}, k_{2} \in R, 0<k_{1}<k_{2} \text {, vimos na secção } 0.5,
\end{aligned}
$$
que o conjunto

$$
K_{0}=\left\{\phi=\left[\begin{array}{c}
\phi_{1} \\
\phi_{2}
\end{array}\right] \in c: 0 \leq k_{1} \phi_{1}(\theta) \leq \phi_{2}(\theta) \leq k_{2} \phi_{1}(\theta) \text {, para }-r \leq \theta \leq 0\right\}
$$

define um cone em $C$. Logo $K=k_{0} \cap \overline{B(0, a)}$ é um cone truncado em $C$, onde $\overline{B(0, a)}$ é a bola fechada de centrozero e raio a em $C$.

$$
\text { Seja } F=\left\{x=\left(x_{1}, x_{2}\right) \in R^{2}: 0 \leq k_{1} x_{1} \leq x_{2} \leq k_{2} x_{1}\right\} \circ \text { cone }
$$
em $R^{2}$, determinado por $k_{1}, k_{2}$. Denotemos por $\xi$, o àngu10 de abertura do cone $F$, isto $\bar{e}, \xi=\operatorname{arctgk}_{2}-\operatorname{arctgk}_{1}$. 
Consideremos a EDFR, autônoma,

$(2.16) \quad \dot{x}(t)=f\left(x_{t}\right)$

de modo que estejam satisfeitas condiçóes que garantam unicidade para $\therefore 0$ problema de valor inicial e continuidade em relaçạo aos dados iniciais e se ja $T(t): C \rightarrow C$ a aplicação solução associada a equação (2.16)

Com as notações acima temos o:

\section{Teorema 2.4}

Suponhamos que:

(i) $C_{a}$ é invariante pela aplicação solução $T(t), t \geq 0$.

(ii) se $\phi \in K \cap C_{a}$ então $S^{1}(a) \subset \underset{t>r}{U} T(t) \phi(0)$.

(iii) $f$ é contínua em $c$ e

$0<|| f(\phi) \|<\frac{a \xi}{r}$, para toda $\phi \in \mathrm{C}_{a}$ tal que $\phi(0) \in \mathrm{F}$.

Então existe uma solução periódica, não constante, da equação $(2.16)$ em $C_{a}$.

\section{Demonstração}

Consideremos os conjuntos

e

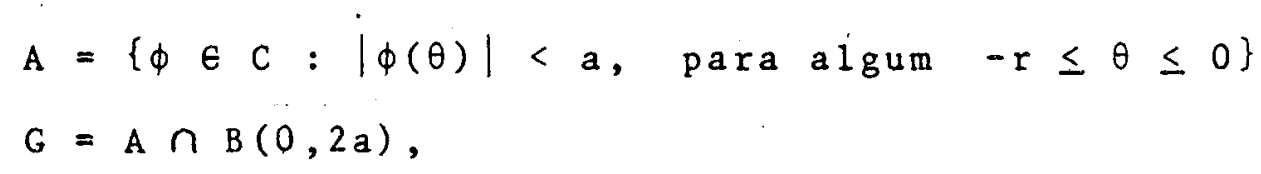

que é aberto e limitado em C. Temos que $\partial G \cap B(0, a)=\emptyset$, pois $B(0, a) \subset G$ e $G \bar{e}$ aberto. Alēm disso, $K \cap \partial G=K \cap C_{a}$, pois, - fato de $C_{a} \subset \partial G$, implica que $K \cap C_{a} \subset k \cap \partial G$ e se $\phi \in k \cap \partial G$, então $\phi$ G, logo $|\phi(\theta)| \geq a$, para todo, $-r \leq \theta \leq 0$; como $\phi \in K$, então $|\phi(\theta)| \leq a$, para todo, $-r \leq \theta \leq 0$, implicando que $|\phi|=a$, logo $\phi \in K \cap C_{a}$.

Consideremos a aplicação, $\tau: k \cap c_{a}+[x, \infty)$ definida 
por $\tau(\phi)=\min \left\{t \geq r: T(t) \phi \in \in K \cap C_{a}\right\}$, para cada $\phi \in \underset{r(L)}{K} \cap C_{a}$, que está bem definida pois se $\phi \in K \cap \mathrm{C}_{a}$, segue de (iii) que existe um menor $t_{0} \geq r$ tal que $T\left(t_{0}\right) \phi(0) \in \partial F$. Por (ii), te mos que $T\left(t_{0}\right) \phi(0) \in F \cap S^{1}(a)$. Mostremos que $\tau(\phi)=t_{0}+r$, is to é, que $T\left(t_{0}+r\right) \phi \in K \cap C_{a}$. De fato, occonjunto

$$
D=\left\{\rho: 0 \leq \rho \leq r \text { e } T\left(t_{0}+\theta\right) \phi(0) \in F \cap S^{1}(a) \text {, para todo } \theta, 0 \leq \theta \leq \rho\right\}
$$

Como $F \cap S^{1}(a)$ é fechado, segue que $D$ também é fechado; assim resta apenas mostrar que $r=\max D$. De fato, se $\bar{\rho}=\max D<r$, existiria uma seqüência, $\left(\theta_{n}\right)$, tal que $\bar{\rho}<\theta_{n} \leq r$ tal que $\theta_{n} \rightarrow \bar{\rho}$, quando $n \rightarrow \infty$ e $T\left(t_{0}+\theta_{n}\right) \phi(0) \notin F \cap S^{l}(a)$ para todo $n \in N$. Assim

(2.17) $\quad\left|\int_{t_{0}}^{t_{0}+\theta_{n}} f\left(x_{s}(\phi)\right) d s\right|>a \xi$.

Da continuidade das soluções da equação (2.16) segue, toman do-se o limite em (2.17), quando $n \rightarrow \infty$, que:

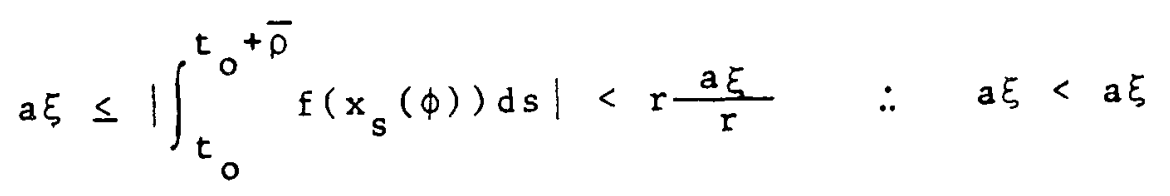

que é um absurdo, logo $m a x D=r$ é portanto $T\left(t_{0}+r\right) \phi \in K \cap C_{a}$.

observemos que $\tau$ é contínua em $K \cap C_{a}$, pois $f(\phi) \neq 0$ para $\phi \in \mathrm{C}_{\mathrm{a}} \operatorname{com} \phi(0) \in \mathrm{F}$ e vale o teorema da continuidade em relação as condições iniciais.

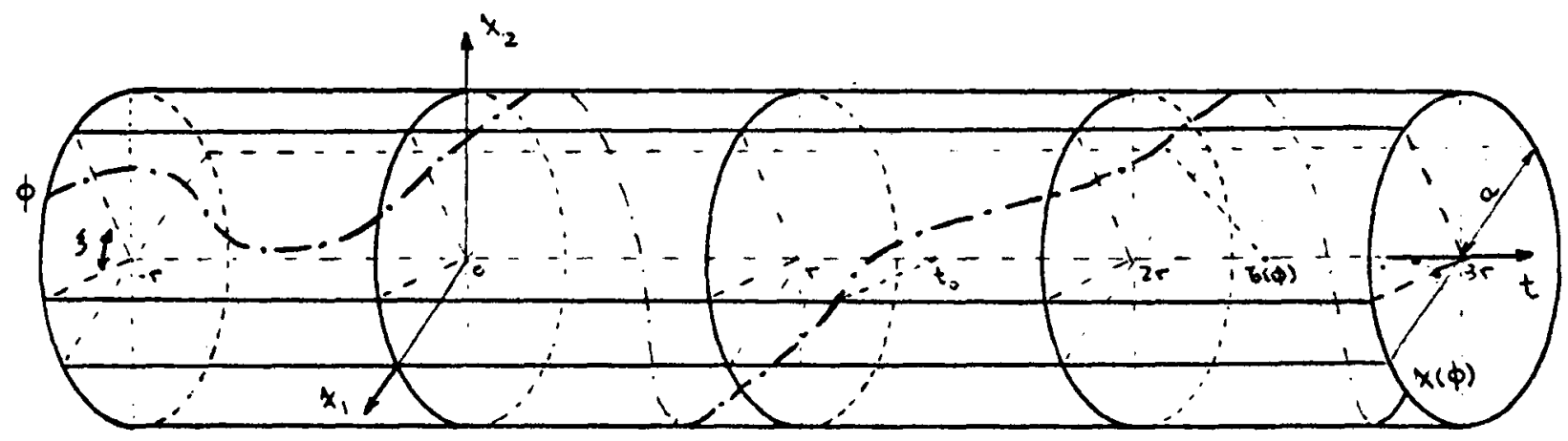


Seja $\tilde{T}: K \cap C_{a} \rightarrow C_{a}$ definida por $\tilde{T}(\phi)=T(\tau(\phi)) \phi$. Temos que $\tilde{\mathrm{T}}\left(\mathrm{K} \cap \mathrm{C}_{\mathrm{a}}\right) \subset \mathrm{K} \cap \mathrm{C}_{\mathrm{a}}$, pois $\phi \in \mathrm{K} \cap \mathrm{C}_{\mathrm{a}}$ então $\tilde{\mathrm{T}}(\phi)=$ $=T(\tau(\phi)) \phi \in K \cap C_{a}$, pela definição da aplicação $\tau$. Além dís so, $\tilde{\mathrm{T}}$ è completamente continua, pois $\mathrm{T}(\mathrm{t}) \phi$ é contínua em $t$, $\phi$ e $\tau(\phi)$ é continua, portanto, $\tilde{T}$ é continua e se $B C K \cap C_{a}$ è 1 imitado, temos que:

(1) $\tilde{\mathrm{T}}(\mathrm{B}) \quad \overline{\mathrm{e}}$ uniformemente limitado, pois $\tilde{\mathrm{T}}(\mathrm{B}) \subset \mathrm{K} \cap \mathrm{C}_{\mathrm{a}}$. Seja $\quad L=\frac{a \xi}{r}$.

(2) $\tilde{\mathrm{T}}(\mathrm{B})$ é equicontinuo, pois dado $\varepsilon>0$, seja $\delta=\frac{\varepsilon}{\mathrm{L}}>0$, então se $\theta_{1}, \theta_{2} \in[-r, 0]$ com $\left|\theta_{1}-\theta_{2}\right|<\delta$ temos que, paratodo $\phi \in \tilde{\mathrm{T}}(\mathrm{B})$, existe $\psi \in \mathrm{B}$ tal que $\tilde{\mathrm{T}}(\psi)=\phi$, logo como $\tau(\phi) \geq r$, $\left|\phi\left(\theta_{1}\right)-\phi\left(\theta_{2}\right)\right|=\left|\tilde{T}(\psi)\left(\theta_{1}\right)-\tilde{T}(\psi)\left(\theta_{2}\right)\right|=$ $=\left|\int_{\tau(\psi)+\theta_{2}}^{\tau(\psi)+\theta_{1}} f\left(x_{s}(\psi)\right) d s\right| \leq$ $\leq\left|\int_{\tau(\psi)+\theta_{2}}^{\tau(\psi)+\theta_{1}}\right| f\left(x_{s}(\psi)\right)|d s|<$ $<\mathrm{L}\left|\theta_{1}-\theta_{2}\right|<\mathrm{L} \delta=\varepsilon$

Logo, pelo Teorema de Ascoli-Arzela segue que $\tilde{T}(B)$ é relativamente compacto, mostrando que $\tilde{\mathrm{T}} \overline{\mathrm{e}}$ completamente contí nua .

Como $K \cap C_{a}=k \cap \partial G$, temos que $\tilde{\mathrm{T}}: \mathrm{k} \cap \partial G \rightarrow \mathrm{k}$ e $\inf \{|\mathrm{T}(\phi)|: \phi \in \mathrm{K} \cap \partial \mathrm{G}\}>0$, pois $\tilde{\mathrm{T}}(\phi) \in \mathrm{K} \cap \mathrm{C}_{\mathrm{a}}, 10 \mathrm{go}$ 
$|\tilde{T}(\phi)|=a>0$. Portanto estamos nas condições do Teorema 0.9 e podemos concluir que $\tilde{\mathrm{T}}$ tem um autovetor $\phi \in \mathrm{K} \cap \partial \mathrm{G}$, isto $\bar{e}$, existe uma constante $\mu$, tal que $\tilde{\mathrm{T}} \phi=\mu \phi$. Como $|\mu \phi|=$ $=|\tilde{T} \phi|=\mathbf{a}=|\phi|$, segue que $|\mu|=1$; mas $\phi$ e $\mu \phi$ pertencem a $k_{0}$, que é um cone, logo $\mu=1$. Portanto. $\phi$ é um ponto fixo de $\tilde{T}$, isto $\bar{e}, \phi=\tilde{T}(\phi)=T(\tau(\phi)) \phi$. Portanto, exis te uma solução periódica, não constante em $\mathrm{C}_{a}$. 


\begin{abstract}
Nós daremos neste capítulo dois exemplos, onde aplicaremos os resultados vistos no Capitulo 2. No primeiro exemplo faremos uma aplicação do Teorema 2.1 , no segundo exemplo uma aplicação do Teorema 2.3 e no terceiro exemplo, do Teorema 2.4 . o exemplo a seguir é uma ligeira modificação de [7; pag. $254]$.
\end{abstract}

\title{
Exemp 10:1
}

Consideremos a equação escalar

(3.1) $\quad \dot{x}(t)=-\alpha x(t-1)[1+g(x(t))]$

onde $\alpha \in R$ e $g: R \rightarrow R$ tal que $g$ tem primeira e segunda derivadas continuas.

Para a equação ( 3.1 ) temos definidas:

$\mathrm{L}(\alpha): C \rightarrow \mathrm{R}$ dada por $\mathrm{L}(\alpha) \phi=-\alpha \phi(-1)$, para $\phi \in \mathrm{C}$

e

$f(\alpha): C \rightarrow R$ dada por $f(\alpha) \psi=-\alpha \psi(-1) g(\psi(0))$, para $\psi \in C$.

Com isto,

$\dot{x}(t)=L(\alpha) x_{t}+f(\alpha) x_{t}$

observemos que $\mathrm{L}(\alpha) \phi$ é linear e continua em $\phi$ e continuamente diferenciāvel em $\alpha$ e a aplicação $f(\alpha) \psi$ é con tinua em $\alpha, \psi$.

Se $F(\alpha, \phi)=-\alpha \phi(-1)[1+g(\phi(0))]$ então podemos escrever a equação ( 3.1$)$ como 


$$
\dot{x}(t)=F\left(\alpha, x_{t}\right)
$$

e $F(\alpha, \phi)$ tem primeira e segunda derivadas continuas em $\alpha, \phi$; $F(\alpha, 0)=0$ para todo $\alpha>0 ; \quad F$ é localmente lipschitziana com relação a $\phi$ e portanto temos unicidade de soluções para equa ção $(3.1)$.

Mostraremos que para $\alpha=\pi / 2$, ocorrer $\vec{a}$ uma bifurcação de Hopf, isto è, existem soluções periödicas da equação $(3.1)$, não nulas, com periódo pröximo a 4, para $\alpha$ suficientemente próxi mo a $\pi / 2$. Para isto precisaremos de informações sobre o compor tamento dos zeros do polinomio característico associado a parte linear da equação (3.1), que è,

(3.2) $\quad \dot{y}(t)=-\alpha y(t-1)$.

A equação característica as sociada a equação (3.2) se$r \bar{a}$

$$
\begin{aligned}
\Delta(\lambda(\alpha))=0, \text { onde } \Delta(\lambda(\alpha)) & =\lambda(\alpha)-\int_{-\mathbf{r}}^{0} \mathrm{dn}(\theta) \mathrm{e}^{\lambda(\alpha) \theta}= \\
& =\lambda(\alpha)-\mathrm{L}(\alpha)\left(\mathrm{e}^{\lambda(\alpha) \cdot)}=\right. \\
& =\lambda(\alpha)+\alpha \mathrm{e}^{-\lambda(\alpha)}
\end{aligned}
$$

e com isto teremos

(3.3) $\quad \lambda(\alpha) \mathrm{e}^{\lambda(\alpha)}=-\alpha$

As informações que necessitaremos estão contidas no se guinte resultado:

\section{Lema 3.1}

Se $0<\alpha<\pi / 2$, cada raiz da equação (3.3) tem parte real negativa. Se $\alpha>e^{-1}$, existe uma raiz, $\lambda(\alpha)=\gamma(\alpha)+i \sigma(\alpha)$ 
da equação (3.3) que é continuamente diferenciável em $\alpha$, e sa tisfaz, $0<\sigma(\alpha)<\pi, \quad \sigma(\pi / 2)=\pi / 2, \quad \gamma(\pi / 2)=0, \gamma^{\prime}(\pi / 2)>0$, $\gamma(\alpha)>0$ para $\alpha>\pi / 2$

\section{Demonstração}

De acordo com [7, pag. 339] todas as raizes da equação $z e^{z}+a e^{z}+b=0$, onde $a, b \in R$ tem parte real negativa se e somente se $a>-1, a+b>0$ e $b<\xi \operatorname{sen} \xi-a \cos \xi$, onde $\xi \quad \vec{e}$ raiz de $\xi=-a t g \xi, \quad 0<\xi<\pi$ se $a \neq 0$ e $\xi=\pi / 2$ se $a=0$.

$$
\begin{aligned}
& \text { Assim escrevendo a equação (3.3) como } \\
& \lambda(\alpha) \mathrm{e}^{\lambda(\alpha)}+\theta \mathrm{e}^{\lambda(\alpha)}+\alpha=0
\end{aligned}
$$

para $0<\alpha<\pi / 2$, concluimos, pelo resultado acima, $\operatorname{Re} \lambda(\alpha)<0$.

Para o restante da prova, definamos $\rho: R \rightarrow R$ por $\rho(\mu)=-\mu \mathrm{e}^{\mu}$. Então $\rho$ é continuamente diferenciävel e $\rho^{\prime}(\mu)=$ $=-(1+\mu) \mathrm{e}^{\mu}$, portanto $\rho^{\prime}(\mu)>0$ para $\mu<-1, \rho^{\prime}(-1)=0$ e $\rho^{\prime}(\mu)<0$ para $\mu>-1$ Logo $\rho$ tem um máximo em $\mu=-1$, dada por $e^{-1}$. Portanto a equação (3.3) não tem raízes reais para $\alpha>e^{-1}$. Se $\alpha>e^{-1}$ e $\lambda=\gamma+i \sigma$ satisfaza equação (3.3) então $\lambda e^{\lambda}=-\alpha$, logo $\gamma+i \sigma=-\alpha e^{-(\gamma+i \sigma)}$ e tomando-se $\gamma=-\mu$, obtemos

$$
\left\{\begin{array}{l}
\mu=\alpha e^{\mu} \cos \sigma \\
\sigma=\alpha e^{\mu} \operatorname{sen} \sigma
\end{array}\right.
$$

ou ainda,

$$
\frac{\mu}{\sigma}=\operatorname{cotg} \sigma \text { e } \alpha=\frac{\sigma}{e_{\operatorname{sen} \sigma}^{\mu}}=\frac{\sigma e^{-\sigma \operatorname{cotg} \sigma}}{\operatorname{sen} \sigma} \mathrm{def} f(\sigma) \text {, }
$$


Observemos que:

(i) $f(\sigma)>0$ para $0<\sigma<\pi$.

(ii) $f(\sigma)$ è diferenciāvel em $\sigma$, para $0<\sigma<\pi$ e

$$
\begin{aligned}
f^{\prime}(\sigma) & =\left\{\operatorname{sen} \sigma\left\{e^{-\sigma \operatorname{cotg} \sigma}+\sigma\left(e^{-\sigma \operatorname{cotg} \sigma}\right)\left[-\operatorname{cotg} \sigma-\sigma\left(-\frac{1}{\operatorname{sen}^{2} \sigma}\right)\right]\right\}-\right. \\
& \left.-\sigma e^{-\sigma \operatorname{cotg} \sigma} \cos \sigma\right\} \frac{1}{\operatorname{sen}^{2} \sigma} .
\end{aligned}
$$

(iii) $\frac{f^{\prime}(\sigma)}{f(\sigma)}=\frac{(1-\sigma \operatorname{cotg} \sigma)^{2}+\sigma^{2}}{\sigma^{2}}>0$ para $0<\sigma<\pi$.

Logo de (i) e (iii), concluímos que $f \vec{e}$ estritamente crescente em $(0, \pi)$. Além disso, $f(\sigma) \rightarrow \infty$ quando $\sigma \rightarrow \pi^{-}$e $f(\sigma) \rightarrow e^{-1}$ quando $\sigma \rightarrow 0^{+}$. Portanto, existe exatamente um valor $\sigma=\sigma(\alpha), \quad 0<\sigma(\alpha)<\pi$, tal que $f(\sigma(\alpha))=\alpha$, se $\alpha>e^{-1}$. Seja $\gamma(\alpha)=-\sigma(\alpha) \operatorname{cotg} \sigma(\alpha)$. As funções $\sigma(\alpha)$ e $\gamma(\alpha)$ são continuamente diferenciāveis em $\alpha$, pois $f \quad e$ continuamente dife renciável, tem inversa em $(0, \pi)$ e $f^{-1}(\alpha)=\sigma(\alpha)$.

$$
\text { Como } f(\pi / 2)=\pi / 2, \text { temos que. } \sigma(\pi / 2)=\pi / 2, \quad \text { logo }
$$

$\gamma(\pi / 2)=0$ ese $\alpha>\pi / 2>e^{-1}$ temosque $\sigma(\alpha)>\pi / 2$, portan to $\gamma(\alpha)>0$ para $\alpha>\pi / 2$.

Derivando ambos os membros da equação (3.3), em relação

a $\alpha$, obtemos

$$
\begin{aligned}
& \lambda^{\prime}(\alpha) \mathrm{e}^{\lambda(\alpha)}+\lambda(\alpha) \lambda^{\prime}(\alpha) \mathrm{e}^{\lambda(\alpha)}=-1 \\
& \text { Calculando em } \alpha=\pi / 2 \text { e usando o fato que } \lambda(\pi / 2)=
\end{aligned}
$$

$=\gamma(\pi / 2)+i \sigma(\pi / 2)=i \pi / 2$, segue que

$$
\gamma^{\prime}(\pi / 2)=\frac{\pi / 2}{1+(\pi / 2)^{2}}>0
$$

concluindo a demonstração do Lema. 
Com isto temos o:

Teorema 3.2

A equação (3.1) tem uma bifurcação de Hopf em $\alpha=\pi / 2$.

\section{Demonstração}

Vimos que $F(\alpha, \phi)$ tem primeira e segunda derivadas con tínuas com relação a $\alpha, \phi$ e $F(\alpha, 0)=0$ para $\alpha>0$ e do Lema 3.1 segue que:

(H1) A EDFR(L( $\pi / 2)$ ) linear autōnoma, tem uma raiz caracterís tica simples, $\lambda(\pi / 2)=i \pi / 2$, imagināria pura e toda raiz característica $\lambda(\alpha) \neq \lambda(\pi / 2), \bar{\lambda}(\pi / 2) \quad$ satisfaz $\lambda(\alpha) \neq \mathfrak{m} \lambda(\pi / 2), \quad \mathfrak{m} \in Z$.

(H2) $\operatorname{Re} \lambda^{\prime}(\pi / 2)=\gamma^{\prime}(\pi / 2)>0$.

Logo estamos nas condições do Teorema 2.1 e com isto po demos concluir que existem constantes $a_{0}, \alpha_{0}, \delta_{0}>0$, funções $\alpha(a)$ e $\omega(a) \in R, \quad x *(a), \quad \omega(a)$-periódica, todas conti nuamente diferenciáveis para $|a|<a_{0}$, tal que $x *(a)$ é solu ção da equação (3.1). Podemos concluir também que o período des tas soluções estão próximas de 4 .

\section{Exemp 10 2}

Consideremos a equação escalar

$$
\dot{x}(t)=-\alpha x(t-1)[1+x(t)]
$$

onde, $\alpha \in \mathbb{R}$.

$$
\text { Se } L(\alpha): C \rightarrow R \text { é dada por } L(\alpha) \phi=-\alpha \phi(-1), f(\alpha): C \rightarrow R
$$

é dada por $f(\alpha) \phi=-\alpha \phi(-1) \phi(0)$ então a equação (3.3) è equiva- 
lente a

$$
\dot{x}(t)=L\left(x_{t}\right)+f\left(x_{t}\right)
$$

A equação característica associada a parte linear de $(3.4) \bar{e}$

(3.5) $\lambda(\alpha) e^{\lambda(\alpha)}=-\alpha$.

Nosso prōximo passo é mostrar que a equação (3.4) tem solução periódica, não constante, para $\alpha>\pi / 2$. Para isto, usa remos o Teorema 2.3 dado no capítulo anterior.

Seja $x(\phi, \alpha)$ a solução da equação (3.1) passando por $\phi$. Se $\phi(0)>-1$ então $x(\phi, \alpha)(t)>-1$ para $t \geq 0$, e não existe $t_{0}>0$ tal que $x(\phi, \alpha)(t)=0$ para $t \geq t_{0}$, a menos que $\phi=0$.

\section{Definição 3.3}

Diremos que os zeros de $x(\phi, \alpha)(t)$ são Zimitados se $x(\phi, \alpha)$ tem somente um nümero finito de zeros positivos.

Com isto temos o:

Lema 3.4

(i) Se $\phi(0)>-1$ e os zeros de $x(\phi, \alpha)(t)$ são limitados então $x(\phi, \alpha)(t) \rightarrow 0$ quando $t \rightarrow \infty$.

(ii) Se $\phi(0)>-1$ então $x(\phi, \alpha)$ é limitada. Além disso, se os zeros de $x(\phi, \alpha)$ não são limitados então qua 1 quer mäximo de $x(\phi, \alpha)(t), t>0$ e menor do que $e^{\alpha}-1$.

(iii) Se $\phi(0)>-1$ e $\alpha>1$, então os zeros de $x(\phi, \alpha)$ não são limitados. 


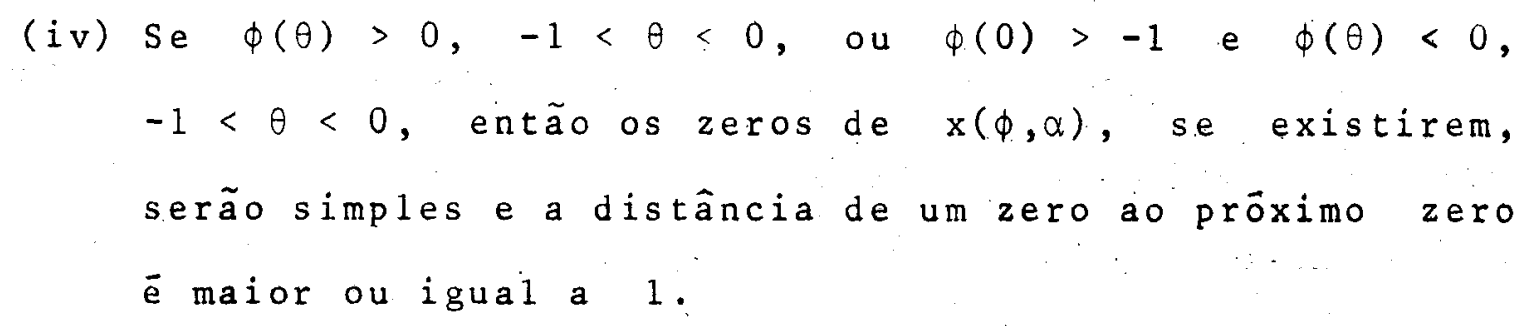

\section{Demonstração}

De (i)

Suponhamos que exista $t_{1}>0$ tal que $x(t)=x(\phi, \alpha)(t)$ tem sinal constante para $t \geq t_{1}-1$, que existe pois os zeros de $x(\phi, \alpha)(t)$ são limitados .

Como $x(t)>-1$, para $t \geq 0$, pois $\phi(0)>-1$, segue que $\dot{x}(t) x(t-1)<0$ para $t \geq t_{1}$. Se $x(t)>0$ para $t \geq t_{1}$ en tão $\dot{x}(t)<0$ para $t \geq t_{1}$ e portanto, $x(t)$ é estritamente decrescente e não se anula para $t \geq t_{1}, \operatorname{logo} x(t) \rightarrow a$ quando $t \rightarrow \infty$, para algum $a \geq 0$. Se $x(t)<0$ para $t \geq t_{1}$ então $\dot{x}(t)>0$ para $t \geq t_{1}$, portanto, $x(t)$ é estritamentecres cente para $t \geq t_{1}$ e não se anula para $t \geq t_{1}, \log 0 x(t) \rightarrow b$ quando $t \rightarrow \infty$, para algum $b \leq 0$. Portanto, $x(t)$ e limitada e aproxima-se, monotonicamente de um ponto limite, quando $t \rightarrow \infty$, isto implica que $\dot{x}(t)$ é limitada e $\dot{x}(t) \rightarrow 0$ quando $t \rightarrow \infty$. Logo, $-\alpha x(t-1)[1+x(t)] \rightarrow 0$ quando $t \rightarrow \infty$, implicando que ou $x(t-1) \rightarrow 0$ ou $x(t) \rightarrow-1$, quando $t \rightarrow \infty$. Como $x(t)+-1$ quan do $t \rightarrow \infty$ segue que $x(t) \rightarrow 0$ quando $t \rightarrow \infty$.

\section{De (ii)}

$$
\text { Temos que } x(t) \text { satisfaz }
$$

(3.6) $1+x(t)=\left(1+x\left(t_{0}\right)\right) \exp \left(-\alpha \int_{t_{0}-1}^{t-1} x(s) d s\right)$ para $t \geq t_{0}$. 
Se os zeros de $x(t)$ são limitados então (i) implica que $x(t)$ é limitada. Se os zeros de $x(t)$ não são limitados então existe uma seqüência de subintervalos $I_{k}$ de $[0, \infty)$, não sobrepostos, tal que $x(t)$ é zero nos extremos de cada subintervalo e tem sinal constante em $I_{k}, k=0,1,2, \ldots$ Logo existe $t_{k} \in I_{k}$ tal que $x\left(t_{k}\right)$ é mäximo ou minimo em $I_{k}$, para cada $k=0,1,2, \ldots ;$ portanto, $0=\dot{x}\left(t_{k}\right)=-\alpha x\left(t_{k}-1\right)\left[1+x\left(t_{k}\right)\right]$, implicando que $x\left(t_{k}-1\right)=0$, pois $x\left(t_{k}\right)>-1$, consequentemente, a equação (3.6) implica, para $t_{0}=t_{k}-1$ e $t=t_{k}$, que $1+x\left(t_{k}\right)<e^{\alpha}$, isto $\bar{e}, \quad x\left(t_{k}\right)<e^{\alpha}-1$ para $k=0,1,2, \ldots, 1$ o go $x(t)<e^{\alpha}-1$ para $t>0$.

\section{De (iii)}

Suponhamos, por absurdo, que os zeros de $x(t)$ são $1 \underline{i}$ mitados, então por (i) segue que $x(t) \rightarrow 0$ quando $t \rightarrow \infty$ e por tanto existe $t_{0}>0$ tal que $\alpha(1+x(t))>1$, para $t \geq t_{0} e$ $x(t)$ tem sinal constante para $t \geq t_{0}$. Assim,

$$
\dot{x}(t) x(t-1)=-\alpha x^{2}(t-1)[1+x(t)]<0 \text { para } t \geq t_{0}+1
$$

e $x(t) \rightarrow 0 ;$ monotonicamente, quando $t \rightarrow \infty$.

$$
\begin{aligned}
& \text { Se } x(t)>0 \text { em }\left[t_{0}, \infty\right) \text { temos que } \\
& x\left(t_{0}+3\right)-x\left(t_{0}+2\right)=\int_{t_{0}+2}^{t_{0}+3} \dot{x}(s) d s<-x\left(t_{0}+2\right)<0
\end{aligned}
$$

portanto $x\left(t_{0}+3\right)<0$, o que é um absurdo, pois $x(t)$ è posi tiva. Um raciocínio análogo para $x(t)<0$ em $\left[t_{0}, \infty\right)$ nos le varā a uma contradição. Portanto os zeros de $x(t)$ não são $1 i-$ mitados . 


\section{De (iv)}

Suponhamos que $x\left(t_{0}\right)=0$ e $x(t)>0$ para $t_{0}-1<t<t_{0}$.

Então para $t_{0}<t<t_{0}+1$ temos que $\dot{x}(t)<0$. Analogamente, se $x(t)<0$ para $t_{0}-1<t<t_{0}$ então $\dot{x}(t)>0$ para $t_{0}<t<t_{0}+1$. Portanto não existirä um zero de $x(t)$ em $\left(t_{0}, t_{0}+1\right]$, logo a distância até o próximo zero, de $x(t)$, será maior ou igual a 1, concluindo a demonstração do Lema.

\section{Consideremos}

$$
K=\{\phi \in C: \phi(\theta) \geq 0,-1<\theta<0, \phi(-1)=0 \text { e } \phi \text { não decrescente }\} \text {. }
$$

Então K é um cone em C.

$$
\begin{aligned}
& \text { Se } \alpha>1 \text { e } \phi \in K, \phi \neq 0 \text { então seja } \\
& z(\phi, \alpha)=\min \{t: x(\phi, \alpha)(t)=0 \text { e } \dot{x}(\phi, \alpha)(t)>0\} .
\end{aligned}
$$

Temos que $z(\phi, \alpha)>1$ e além disso do Lema 3.4 segue que $x(\phi, \alpha)(t)$ é positiva e não decrescente em $(z(\phi, \alpha), z(\phi, \alpha)+1)$.

Definamos $\tau:(K \backslash\{0\}) \times(1, \infty) \rightarrow(0, \infty)$ por $\tau(\phi, \alpha)=$ $=z(\phi, \alpha)+1$. Então $\tau$ é continua e temos o:

\section{Lema 3.5}

A ap1icação $\tau(\phi, \alpha)$, definida acima, $\vec{e}$ completamente contínua.

\section{Demonstração}

Afirmamos que uma solução $x=x(\phi, \alpha)$, para $\phi \in K$ e $\alpha>1$, não pode demorar um tempo maior do que 2 para tornar-se negativa, pois se $x(1)=\eta>0$, pela equação (3.6), teremos que

$$
x(2) \leq(1+n) \mathrm{e}^{-\alpha \eta}-1<0
$$


pois $h(n)=(1+n) e^{-\alpha \eta}-1$ satisfaz:

$$
h(0)=0 \text { e } h^{\prime}(\eta)=(-\alpha-\alpha \eta+1) e^{-\alpha \eta} \text {. }
$$

Como $\alpha>1$ e $n>0$ temos que $h^{\prime}(n)<0, \log 0 h(n)<0$ pa ra $\eta>0$.

$$
\therefore \mathrm{x}(2)<0 \text {. }
$$

Para cada $B \subset K$ limitado, $\phi \in B, \alpha>1$ seja $t_{0}(\phi, \alpha) \leq 3$, o ponto onde $x=x(\phi, \alpha)$ tem um minimo. Como $t_{0}(\phi, \alpha) \geq 1, \quad 0$ conjunto $H(\alpha)=\overline{U_{\phi \in B} x_{t_{0}}(\phi, \alpha)}(\phi, \alpha) \subset C \quad \bar{e}$ compacto, pois ê uniformemente limitado e equicontinuo.

Temos que $\mathrm{H}(\alpha) \subset \mathrm{K}_{1}=\{\phi \in \mathrm{C}:-1<\psi(\theta) \leq 0,-\mathrm{r} \leq \theta \leq 0$ e $\psi$ não crescente\}.

Definindo $\tau_{1}:\left(k_{1} \backslash\{0\}\right) \times(1, \infty) \rightarrow(0, \infty)$ por $\tau_{1}(\alpha)=\min \{t>0: x(\psi, \alpha)(t)=0\}$, temos que $\tau_{1} \bar{e}$ continua. Se provarmos que $\tau_{1}(H(\alpha) \backslash\{0\}, \alpha)$ è limitado para cada $\alpha \in(1, \infty)$ então teremos que $\tau(B \backslash\{0\}, \alpha)$ serä 1 imitado para cada $\alpha \in(1, \infty)$. Como $H(\alpha) \backslash\{0\} \subset H(\alpha)$ então

$$
\tau_{1}(H(\alpha) \backslash\{0\}, \alpha) \subset \tau_{1}(H(\alpha), \alpha),
$$

mas $H(\alpha)$ é compacto e $\tau_{1}$ é continua, portanto $\tau_{1}(H(\alpha), \alpha)$ é limitado para cada $\alpha \in(1, \infty)$. Logo $\tau(B \backslash\{0\}, \alpha)$ è 1imita do para cada $\alpha \in(1, \infty)$.

Se $A \subset(1, \infty)$ é 1 imitado e fechado então A è compac to e como $\tau$ é contínua segue que $\tau(\phi, A)$ è limitado em $R$. Logo $\tau$ é completamente continua.

$$
\begin{aligned}
& \text { Para } \alpha>1, \text { definamos } \\
& \mathrm{A}(\alpha) \phi= \begin{cases}0 & \mathrm{~A}(\alpha) \\
0 & \text { se } \phi=0 \\
\mathrm{x}_{\tau(\phi, \alpha)}(\phi, \alpha) & \text { se } \phi \neq 0\end{cases}
\end{aligned}
$$


Então $A(x) K \subset K \quad$ e além disso temos o:

Lema 3.6

A aplicação $A(\alpha): K \rightarrow K$ definida acima é completamen te continua.

\section{Demonstração}

Temos que $A(\alpha): K \rightarrow K$ é continua pois $\tau(\phi, \alpha)$ é con tínua e as soluções da equação (3.4) dependem continuamente em relação as condiçōes iniciais.

Para mostrarmos que $A(\alpha)$ leva conjuntos limitados em conjuntos relativamente compacto usaremos o Teorema de Ascoli-Arzela.

Se BCK é limitado então a parte (ii) e (iii) do Lema 3.4 implica que:

$$
\begin{aligned}
& |A(\alpha) \phi|=\left|x_{\tau(\phi, \alpha)}(\phi, \alpha)\right|<e^{\alpha}-1 \text {, pois } \alpha>1 \text {. } \\
& \text { Portanto A( } \alpha) \text { B é uniformemente limitado. } \\
& \text { Além disso A( } \alpha) B \text { é equicontínuo, pois se } \\
& \mathrm{x}_{\tau(\phi, \alpha)}(\phi, \alpha) \in \mathrm{A}(\alpha) B, \text { temos que } \\
& D_{\theta} x_{\tau(\phi, \alpha)}(\phi, \alpha)<\alpha\left(e^{\alpha}-1\right)\left[1-\left(e^{\alpha}-1\right)\right],
\end{aligned}
$$

logo a derivada dos elementos de $A(\alpha) B$ são uniformemente 1 im 1 tadas implicando que $A(\alpha) B$ é equicontínuo. Logo $A(\alpha) B$ é rela tivamente compacto, mostrando que $A(\alpha)$ é completamente continua.

Seja $\lambda(\alpha)$ a raiz da equação (3.5) dada pelo Lema 3.1, $\quad C=P_{\lambda(\alpha)} \oplus Q_{\lambda(\alpha)}$ a decomposição de $C$ pelo autovalor $\lambda(\alpha)$, 
$\pi_{\lambda(\alpha)}$ a projeção de C em $P_{\lambda(\alpha)}$. Então temos o:

\section{Lema 3.7}

Se Jo è um subconjunto compacto de $(1, \infty)$ então

$\mu=\inf \left\{\left|\pi_{\lambda(\alpha)} \phi\right|: \phi \in \mathrm{K},|\phi|=1\right.$ e $\left.\alpha \in \mathrm{J}_{0}\right\}>0$

\section{Demonstração}

Sejam $\lambda=\lambda(\alpha)$ a solução da equação (3.3) dada pelo Lema 3.1, $\phi(\theta)=\frac{e^{\lambda \theta}}{1+\lambda}, \quad-1 \leq \theta \leq 0$ e $\quad \psi(s)=e^{-\lambda s}, \quad 0 \leq s \leq 1$ e consideremos $\Phi=(\phi, \bar{\phi})$ e $\Psi=(\psi, \bar{\psi})$.

A equação adjunta formal da equação (3.2) é $\dot{\mathbf{z}}(\mathrm{t})=\alpha z(t+1)$.

Temos que:

$$
\begin{aligned}
(\psi, \phi) & =\psi(0) \phi(0)-\int_{-1}^{0} \mathrm{dn}(\theta) \int_{0}^{\theta} \psi(\xi-\theta) \phi(\xi) \mathrm{d} \xi= \\
& =\psi(0) \phi(0)-\alpha \int_{-1}^{0} \psi(\xi+1) \phi(\xi) \mathrm{d} \xi
\end{aligned}
$$

e $(\Psi, \Phi)=I_{2}=$ identidade de ordem 2 .

Portanto, para $n \in C$ temos que $\pi_{\lambda}{ }^{n}=\Phi(\Psi, \eta)$.

Agora,

$\inf \left\{\left|\pi_{\lambda} n: \eta \in K,\right| \eta \mid=1, \alpha \in J_{0}\right\}=$

$=\inf \left\{|\Phi(\Psi, \eta)|: \eta \in \mathrm{K},|\eta|=1, \alpha \in \mathrm{J}_{0}\right\}>0$,

pois

$$
(\Psi, \eta)=\left[\begin{array}{l}
(\psi, \eta) \\
(\bar{\psi}, \eta)
\end{array}\right]
$$


e como $(\psi, \eta)$ e $(\bar{\psi}, \eta)$ são complexos conjugados, basta olhar mos para $(\Psi, \eta)$. Se $\eta \in K$ e $|\eta|=1$ então $n(-1)=0$, $n$ é não decrescente, contínua, logo $\eta(0)=1$ e portanto temos

$$
(\psi, n)=\psi(0) \eta(0)-\alpha \int_{-1}^{0} \psi(\xi+1) \eta(\xi) d \xi=R(n)+i I(n),
$$

onde $R(\eta)=1-\alpha \int_{-1}^{0} e^{-\gamma(\xi+1)} \cos \sigma(\xi+1) \eta(\xi) d \xi \quad$ e

$$
I(n)=\alpha-\int_{-1}^{0} e^{-\gamma(\xi+1)} \operatorname{sen} \sigma(\xi+1) n(\xi) d \xi
$$

$\operatorname{com} . \lambda=\gamma+i \sigma$.

Visto que $J_{0} e-$ compacto, existe $a>0$ tal que $0<a<\sigma=\sigma(\alpha)<\pi-a$ para $\alpha \in \mathrm{J}_{0}$.

Suponhamos, por absurdo, que exista uma seqüência $\left(n_{n}\right)$, $n_{n} \in K, \operatorname{com} n_{n}(0)=1$ e uma seqüềncia $\left(\alpha_{n}\right)$ em $J_{0}$ tal que $\left(\psi, n_{n}, \alpha_{n}\right) \stackrel{\text { def }}{=}\left(\psi, n_{n}\right) \rightarrow 0$ quando $n \rightarrow \infty$. Podemos supor que $\alpha_{n} \rightarrow \beta$ quando $n \rightarrow \infty$, pois $J_{0} \bar{e}$ compacto. Como $I\left(n_{n}\right) \rightarrow 0$ temos que $\eta_{n}(\theta) \rightarrow 0$ quando $n \rightarrow \infty$ para todo $-1 \leq \theta \leq 0$. As sim $R\left(n_{n}\right)+1$ quando $n \rightarrow \infty$, mas isto contraria o fato de $R\left(n_{n}\right) \rightarrow 0$ quando $n \rightarrow \infty$, logo não existe $\left(n_{n}\right)$ em $k$, com $\eta_{n}(0)=1$ e $\left(\alpha_{n}\right)$ em $J_{0}$ tal que $\left(\psi, n_{n}, \alpha_{n}\right) \rightarrow 0$ quando $n \rightarrow \infty$. Portanto $\inf \left\{|(\Psi, \eta)|: \eta \in K,|\eta|=1, \alpha \in J_{0}\right\}>0$ e o Lema es tà provado.

\section{Lema 3.8}

Existe $M>0$ tal que $A(\alpha) \phi=\lambda \phi$, para todo $\phi \in K n s_{M}$ então $\quad \lambda<1$. 


\section{Demonstração}

Suponhamos, por absurdo, que isto não ocorra, isto é, para todo $M>0$, existe $\phi \in K \cap S_{M}$ tal que $A(\alpha) \phi=\lambda \phi$, para algum $\lambda \geq 1$. Tomando-se $M>e^{\alpha}-1$, teremos que

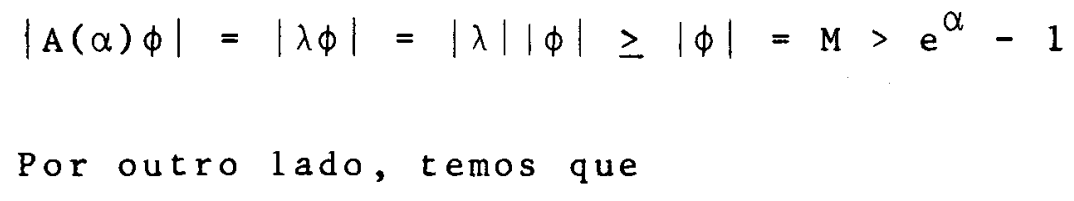

para toda $\phi \in K, \quad$ que é um absurdo coma expressão acima, e - Lema está provado.

Estamos nas condições do Teorema 2.3 e com isto demons trar o:

\section{Teorema 3.9}

Se $\alpha>\pi / 2$ a equação $(3,4)$ tem uma solução periödica não nula, com condição inicial em $K \backslash\{0\}$.

\section{observação 3.10}

Outros exemplos, incluindo o exemplo acima, podem ser encontrados em [7; pag. 260-261].

\section{Exemp 10 3}

Consideremos a equação

(3.5) $\left\{\begin{array}{l}\dot{x}(t)=y(t) g\left(x_{t}, y_{t}\right)+h_{1}\left(x_{t}, y_{t}\right) \\ \dot{y}(t)=-x(t) g\left(x_{t}, y_{t}\right)+h_{2}\left(x_{t}, y_{t}\right)\end{array}\right.$

onde $g, h_{1}, h_{2}: \bar{C} \times \bar{C} \rightarrow R$ são continuas com $\bar{C}=c([-r, 0], R)$. 
Seja $f: C \rightarrow R^{2}$ dada por

$$
f(\phi)=\left[\begin{array}{c}
\phi_{2}(0) g\left(\phi_{1}, \phi_{2}\right)+h_{1}\left(\phi_{1}, \phi_{2}\right) \\
-\phi_{1}(0) g\left(\phi_{1}, \phi_{2}\right)+h_{2}\left(\phi_{1}, \phi_{2}\right)
\end{array}\right]
$$

onde $\phi=\left[\begin{array}{c}\phi_{1} \\ 1 \\ \phi_{2}\end{array}\right] \in C=C\left([-r, 0], R^{2}\right)$.

$$
\text { Então se } x(t)=\left[\begin{array}{c}
x(t) \\
y(t)
\end{array}\right] \text {, temos que a equação }
$$

é equivalente a equação

$$
\dot{X}(t)=f\left(x_{t}\right)
$$

Sejam $0<k_{1}<k_{2}$ e $F=\left\{x=\left(x_{1}, x_{2}\right) \in R^{2}: 0 \leq k_{1} x_{1} \leq x_{2} \leq k_{2} x_{1}\right\} \circ$ cone em $R^{2}$ determinado por $k_{1}, k_{2}$, cujo ângulo de abertura é $\xi=\operatorname{arctgk}_{2}-\operatorname{arctgk}_{1}, \operatorname{com} 0<\xi<\pi$.

Seja

$$
\mathrm{K}_{0}=\left\{\phi:\left[\begin{array}{l}
\phi_{1} \\
\phi_{2}
\end{array}\right] \in \mathrm{C}: \mathrm{k}_{1} \phi_{1}(\theta) \leq \phi_{2}(\theta) \leq \mathrm{k}_{2} \phi_{1}(\theta)\right\}
$$

um cone em $C$ e $K=K_{0} \cap \overline{B(0, a)}$ o cone truncado pela bola fechado de centrozero e raio a em C. (a>0).

Suponhamos que:

(1) Se $\phi \in C_{a}$ então $h_{1}(\phi)=h_{2}(\phi)=0$, para algum a $>0$. 
(2) Para toda $\phi \in C_{a} \operatorname{com} \phi(0) \in F$ temos que $|g(\phi)|<\frac{\xi}{2 r}=M$.

(3) Existe $\varepsilon>0$ tal que $|\mathrm{g}(\phi)|>\varepsilon$ para toda $\phi \in \mathrm{C}_{\mathrm{a}}$. Observemos que com estas condições:

(i) Se $\phi \in \mathrm{C}_{\mathrm{a}} \operatorname{com} \phi(0) \in \mathrm{F}$ então de (1) e (2) segue que

$$
\begin{aligned}
|\mathrm{f}(\phi)| & =\left|\phi_{2}(0) \mathrm{g}(\phi)\right|+\left|\phi_{1}(0) \mathrm{g}(\phi)\right| \leq \\
& \leq 2|\phi||g(\phi)|<2 a M
\end{aligned}
$$

Além disso, como $\phi(0) \in \mathrm{s}^{1}($ a $)$ temos

$$
|f(\phi)|=\left|\phi_{2}(0) g(\phi)\right|+\left|\phi_{1}(0) g(\phi)\right|>0 \text {. }
$$

Logo $0<|f(\phi)|<\frac{a \xi}{r}$ se $\phi \in C_{a} \operatorname{com} \phi(0) \in F$.

(ii) $C_{a}$ é invariante pela aplicação solução, $T(t)$, associada a equação (3.5), pois se

$x(t)=\left[\begin{array}{l}x(t) \\ y(t)\end{array}\right]$

é uma solução da equação (3.5) temos que:

$x(t) \dot{x}(t)+y(t) \dot{y}(t)=x(t) h_{1}\left(x_{t}\right)+y(t) h_{2}\left(x_{t}\right)$.

Logo, por (1), para toda condição inicial em $\mathrm{C}_{a}$ tere mos

$x(t) \dot{x}(t)+y(t) \dot{y}(t)=0$, assim $x^{2}(t)+y^{2}(t)=a^{2}$.

Portanto toda solução com condição inicial em $\mathrm{C}_{a}$, per manecerä em $\mathrm{C}_{\mathrm{a}}$. 
(iii) Se $\phi \in K \cap \mathrm{C}_{\mathrm{a}}$ então $\mathrm{T}(\mathrm{t}) \phi(0)$ cobre $\mathrm{S}^{1}(\mathrm{a})$, para $t \geq r$, pois se $\phi \in K \cap \mathrm{C}_{\mathrm{a}}$ temos que $T(t) \phi(0)=\left[\begin{array}{l}x(\phi)(t) \\ y(\phi)(t)\end{array}\right] \in S^{1}(a)$,

pois $C_{\text {a }}$ é $T(t)$-invariante. De (3) segue que ou $g(\phi)>\varepsilon$ ou $g(\phi)<-\varepsilon$, assim $\dot{x}(t)$ tem o mesmo sinal de $y(t)$ e $\dot{y}(t)$ tem sinal conträrio de $x(t)$, ou vice-versa e tanto $\dot{x}(t)$ como $\dot{y}(t)$ não tendem a zero quando $t \rightarrow \infty$, assim $\mathrm{T}(\mathrm{t}) \phi(0)$ percorre $\mathrm{S}^{1}(\mathrm{a})$ horärio ou anti-horário e portanto

$$
S^{1}(a) \subset \underset{t>r}{U} T(t) \phi(0)
$$

Logo as hipóteses do Teorema 2.4 estão satisfeitas e podemos concluir que existe uma solução periödica, não constan te, da equação (3.5) em $\mathrm{C}_{\mathrm{a}}$.

\section{Observação 3.11}

E fácil exibir funções $g, h_{1}$ e $h_{2}$ nas condições especificadas acima. Por exemplo:

$$
\mathrm{g}: \mathrm{C} \rightarrow \mathrm{R} \text { dada por } \mathrm{g}(\phi)=\frac{\xi}{2 \mathrm{a}}\left|\int_{-\mathrm{r}}^{\mathrm{O}} \phi(\mathrm{s}) \mathrm{d} \mathrm{s}\right|+\varepsilon, \phi \in \mathrm{C}
$$

e

$$
\mathrm{h}_{1}, \mathrm{~h}_{2}: \mathrm{C} \rightarrow \mathrm{R} \text { dadas: } \mathrm{h}_{1}(\phi)=\mathrm{h}_{2}(\phi)=\left|\phi_{1}\right|^{2}+\left|\phi_{2}\right|^{2}-\mathrm{a}^{2}
$$

onde

$$
\phi=\left[\begin{array}{c}
\phi_{1} \\
\phi_{2}
\end{array}\right] \in \mathrm{C} \quad \text { e } \quad \mathrm{a}>0
$$

A idéia do exemplo 3 baseia-se num exemplo contido em [2; pag. 31]. 
[1] - Banks, H.T. - The Representation of Solutions of Linear Differential Equations. Journal of Diff. Eq. 5(1966), 399-410.

[2] - Baroni, R.L.S. - Tese de Doutorado apresentada ao ICMSC-USP, 1983.

[3] - Butzer, P.L. and Berens, H. - Semi-groups of Operators and Approximation. Springer-Verlag, New York (1967).

[4] - Cameron, R.H. and Martins, W.T. - Anunsymetric Fubini Theorem. Bull. Amer. Math. Soc., 47(1971), 121-125.

[5] - Hale, J.K. - Ordinary Differential Equations. Wiley, (1969).

[6] - Hale, J.K. - Functional Differential Equations. Applied Mathematics Sciences, Vol. 3, Springer-Verlag, New York, (1971).

[7] - Hale, J.K. - Theory of Functional Differential Equations. Springer-Verlag, New York Inc., (1977).

[8] - Hale, J.K.; Magalhães, L.T; Oliva, W.M. - An Introduction to Infinite Dimensional Dynamical Systems - Geometric Theory. Springer-Verlag, (1984). Applied Math. Series, 47.

[9] - Kato, T. - Perturbation Theory for Linear Operators. Springer-Verlag, New York, (1976).

[10] - Kolmogorov, A.N. y Folmin, S.V. - Elementos de la Teoria de Funciones y del Analisis Funcional. Editorial Mir-Moscu, (1972).

[11] - Nussbaum, R. - Periodic Solutions of Some Nonlinear Autonomous Functional Differential Equations. Ann. Math. Pura Apl. 10(1974), 263-306.

[12] - Onuchic, N. - Equações Diferenciais com Retardamento, $8 .^{\circ}$ Colóquio Brasileiro de Matemätica, (1971).

[13] - Pazy, A. - Semi-groups of Linear Operators and Applications to Partial Differential Equations. Lecture Note \#10. University of Maryland (197.4).

[14] - Taylor, A.L. - Introduction to Functional Analysis. John Wiley \& Sons, Inc. New York (1958). 